\title{
Inspiral-merger-ringdown waveforms of spinning, precessing black-hole binaries in the effective-one-body formalism
}

\author{
Yi Pan, ${ }^{1}$ Alessandra Buonanno, ${ }_{1}^{1}$ Andrea Taracchini, ${ }^{1}$ Lawrence E. Kidder, ${ }^{2}$ Abdul H. Mroué, ${ }^{3}$ \\ Harald P. Pfeiffer, ${ }^{3,4}$ Mark A. Scheel, ${ }^{5}$ and Béla Szilágyi ${ }^{5}$ \\ ${ }^{1}$ Department of Physics, Maryland Center for Fundamental Physics \& Joint Space-Science Institute, \\ University of Maryland, College Park, Maryland 20742, USA \\ ${ }^{2}$ Center for Radiophysics and Space Research, Cornell University, Ithaca, New York 14853, USA \\ ${ }^{3}$ Canadian Institute for Theoretical Astrophysics, 60 St. George Street, \\ University of Toronto, Toronto, Ontario M5S 3H8, Canada \\ ${ }^{4}$ Canadian Institute for Advanced Research, 180 Dundas St. West, Toronto, Ontario M5G 1Z8, Canada \\ ${ }^{5}$ Theoretical Astrophysics 350-17, California Institute of Technology, Pasadena, California 91125, USA
}

(Received 1 August 2013; published 2 April 2014)

\begin{abstract}
We describe a general procedure to generate spinning, precessing waveforms that include inspiral, merger, and ringdown stages in the effective-one-body (EOB) approach. The procedure uses a precessing frame in which precession-induced amplitude and phase modulations are minimized, and an inertial frame, aligned with the spin of the final black hole, in which we carry out the matching of the inspiral-plunge to merger-ringdown waveforms. As a first application, we build spinning, precessing EOB waveforms for the gravitational modes $\ell=2$ such that in the nonprecessing limit those waveforms agree with the EOB waveforms recently calibrated to numerical-relativity waveforms. Without recalibrating the EOB model, we then compare EOB and post-Newtonian precessing waveforms to two numerical-relativity waveforms produced by the Caltech-Cornell-CITA collaboration. The numerical waveforms are strongly precessing and have 35 and 65 gravitational-wave cycles. We find a remarkable agreement between EOB and numerical-relativity precessing waveforms and spins' evolutions. The phase difference is $\sim 0.2 \mathrm{rad} \mathrm{rad}$ at merger, while the mismatches, computed using the advanced-LIGO noise spectral density, are below $2 \%$ when maximizing only on the time and phase at coalescence and on the polarization angle.
\end{abstract}

DOI: 10.1103/PhysRevD.89.084006

\section{INTRODUCTION}

An international network of gravitational-wave $(\mathrm{GW})$ detectors operating in the frequency band $10-10^{3} \mathrm{~Hz}$ exists today. It is composed of the LIGO detectors in Hanford, Washington, and Livingston, Louisiana, in the United States; the French-Italian Virgo detector [1]; and the British-German GEO600 detector [2]. Those detectors have collected and analyzed data for several years. Since 2010 they have been shut down to be upgraded to the advanced LIGO and Virgo configurations [3]. The design sensitivity for advanced detectors, which is planned to be achieved by 2019 [4], will be a factor of 10 better than the one of the initial detectors. This improvement implies an increase in the event rates of coalescing binary systems of (roughly) one thousand, thus making very likely the first detection of gravitational waves [4] with the advanced detector network. Furthermore, efforts to build a gravitational-wave detector in space are underway [5].

Binary systems composed of compact objects, such as black holes and neutron stars (compact binaries for short) are the most promising sources for ground-based GW detectors. The signal detection and interpretation are based on a matched-filtering technique, where the noisy detector output is cross-correlated with a bank of theoretical templates.
PACS numbers: 04.25.D-, 04.25.dg, 04.25.Nx, 04.30.-w

Fueled by numerical-relativity (NR) simulations, there has been substantial progress in building and validating accurate templates for the inspiral, merger, and ringdown stages of nonprecessing ${ }^{1}$ black-hole (BH) binaries [6-18] (see also Ref. [19], where several analytical templates have been compared to simulations produced by the numericalrelativity and analytical-relativity (NRAR) collaboration). Despite this progress, template modeling for generic, spinning $\mathrm{BH}$ binaries is far from being complete. In this paper, we focus on $\mathrm{BH}$ binary systems moving on quasispherical orbits, i.e., generic precessing orbits that are circularized and shrunk by radiation reaction.

During the last several years, the post-Newtonian (PN) formalism, which expands the Einstein equations in powers of $v / c$ ( $v$ being the characteristic velocity of the binary and $c$ the speed of light), has extended the knowledge of the dynamics and gravitational waveform for quasispherical orbits through 3.5PN $[20,21]$ and $2 \mathrm{PN}[22]$ order, respectively. Precession-induced modulations in the phase and amplitude of gravitational waveforms become stronger as the opening angle between the orbital angular momentum

\footnotetext{
${ }^{1}$ Here, nonprecessing means that the $\mathrm{BH}$ spins are either zero or aligned/antialigned with the orbital angular momentum.
} 
and the total angular momentum of the binary increases. Compact binaries with large mass ratios can have larger opening angles than those with comparable masses.

Pioneering studies aimed at understanding and modeling precession effects in inspiraling compact binaries were carried out in the mid 1990s [23,24]. As GW detectors came online in early 2000, it became more urgent to develop template families for spinning, precessing binaries in which precession-induced modulations were incorporated in an efficient way, reducing also the dimensionality of the parameter space. In 2003, Buonanno, Chen, and Vallisneri [25] introduced the precessing convention and proposed a template family for precessing binaries in which precessional effects are neatly disentangled from nonprecessing effects in both the amplitude and phase evolutions. They also showed that the (restricted) waveform in the frame that precesses following the precessing convention takes the simple nonprecessing form and that strong correlations exists between the nonprecessing phase evolution and spin parameters, notably the opening angle between the spin and orbital angular momentum (see Fig. 15 in Ref. [25]). This correlation was further studied and modeled in Ref. [26]. More recently, Ref. [27] explicitly demonstrated the agreement between nearly nonprecessing waveforms in the precessing frame and exact nonprecessing waveforms generated by neglecting the spin components in the orbital plane. The precessing convention was initially introduced to model phenomenological or detection template families [25], and then it was extended to physical templates of single-spin binary systems in Refs. [26,28]. In the past few years, geometric methods have been developed to construct preferred precessing reference frames [29-32] for numerical or analytical waveforms, achieving a similar disentanglement of precessional effects. Waveforms decomposed in such frames exhibit relatively smooth amplitude and phase evolutions and are well approximated by nonprecessing waveforms [27,33].

Here, we use the effective-one-body (EOB) formalism [34-37] to model precessing inspiral, merger, and ringdown waveforms. The basic idea of the EOB approach is to map by a canonical transformation the conservative dynamics of two compact objects of masses $m_{1}$ and $m_{2}$ and spins $\boldsymbol{S}_{1}$ and $\boldsymbol{S}_{2}$ into the dynamics of an effective particle of mass $\mu=m_{1} m_{2} /\left(m_{1}+m_{2}\right)$ and spin $S_{*}$ moving in a deformed Kerr metric with mass $M=m_{1}+m_{2}$ and spin $S_{\text {Kerr }}$, the deformation parameter being the symmetric mass ratio $\nu=\mu / M$. In the mid 2000s, Buonanno, Chen, and Damour [38] modeled EOB inspiral, merger, and ringdown waveforms including for the first time spinning, precessing effects.

In this paper we build on Refs. [25,38], and also on the most recent analytical work [39-41] and the work at the interface between numerical and analytical relativity $[11,13]$, and develop a general procedure to generate spinning, precessing waveforms in the EOB approach.
The procedure employs the precessing convention of Ref. [25] and an inertial frame aligned with the spin of the final BH. As a first application, we construct spinning, precessing waveforms that contain only the $\ell=2$ gravitational mode and reduce to the nonprecessing waveforms calibrated to NR waveforms [42-45] in Ref. [13]. We compare these EOB precessing waveforms to Taylorexpanded PN waveforms and to two NR waveforms recently produced by the Caltech-Cornell-CITA collaboration [46].

The paper is organized as follows. In Sec. II we discuss the main coordinate frames that are used to describe precessing waveforms and their physical characteristics. We also review different proposals that have been suggested in the analytical and numerical-relativity communities for the precessing source frame, in which precession-induced modulations in the waveform's phase and amplitude are minimized. We also study how spin components parallel to the orbital plane affect the energy flux and multipolar waveforms. In Sec. III we build EOB precessing waveforms using a precessing source frame aligned with the Newtonian orbital angular momentum and an inertial frame aligned with the direction of the final $\mathrm{BH}$ spin. In Sec. IV we compare EOB precessing waveforms computed in different precessing source frames and carry out comparisons between EOB, Taylor-expanded PN, and NR precessing waveforms. Section V summarizes our main conclusions and future work.

\section{MODELING PRECESSING WAVEFORMS}

\section{A. Conventions and inertial frames}

Throughout the paper we adopt geometric units $G=$ $c=1$ and the Einstein summation convention, unless otherwise specified. The masses of the BHs are $m_{1}$ and $m_{2}$, and we choose $m_{1} \geq m_{2}$. The total mass, mass ratio, and symmetric mass ratio are $M=m_{1}+m_{2}, q=m_{1} / m_{2}$, and $\nu=m_{1} m_{2} / M^{2}$, respectively. The position, linear momentum, and spin vectors of the BHs are $\boldsymbol{r}_{i}(t), \boldsymbol{p}_{i}(t)$, and $\boldsymbol{S}_{i}(t)=\chi_{i} m_{i}^{2} \hat{\boldsymbol{S}}_{i}$, where $i=1,2$ and $\chi_{i}$ is the dimensionless spin magnitude. In the EOB framework, we solve the time evolution of the relative (rescaled) position vector $\boldsymbol{r}(t) \equiv\left(\boldsymbol{r}_{1}(t)-\boldsymbol{r}_{2}(t)\right) / M$, the center-of-mass-frame (rescaled) momentum vector $\boldsymbol{p}(t) \equiv \boldsymbol{p}_{1}(t) / \mu=-\boldsymbol{p}_{2}(t) / \mu$, and the spins variables $S_{1}(t)$ and $S_{2}(t)$.

We start with an arbitrary orthonormal basis $\left\{\boldsymbol{e}_{x}, \boldsymbol{e}_{y}, \boldsymbol{e}_{z}\right\}$. Without loss of generality, we align the initial relative position vector $\boldsymbol{r}_{0}$ with $\boldsymbol{e}_{x}$ and the initial orbital orientation $\left[\hat{\boldsymbol{L}}_{N}\right]_{0} \equiv \hat{\boldsymbol{L}}_{N}(0) \equiv \boldsymbol{r}_{0} \times \dot{\boldsymbol{r}}_{0} /\left|\boldsymbol{r}_{0} \times \dot{\boldsymbol{r}}_{0}\right|$ with $\boldsymbol{e}_{z}$, where we indicate with an overdot the time derivative and $\dot{\boldsymbol{r}}_{0}=\dot{\boldsymbol{r}}(0)$ is the initial relative velocity. The initial spin directions are specified by the spherical coordinates $\theta_{1}^{S}, \phi_{1}^{S}, \theta_{2}^{S}$, and $\phi_{2}^{S}$ associated with this basis.

In the nonprecessing case, $\hat{\boldsymbol{L}}_{N}$ is a constant, and it is natural to choose a source frame described by the (unit) 


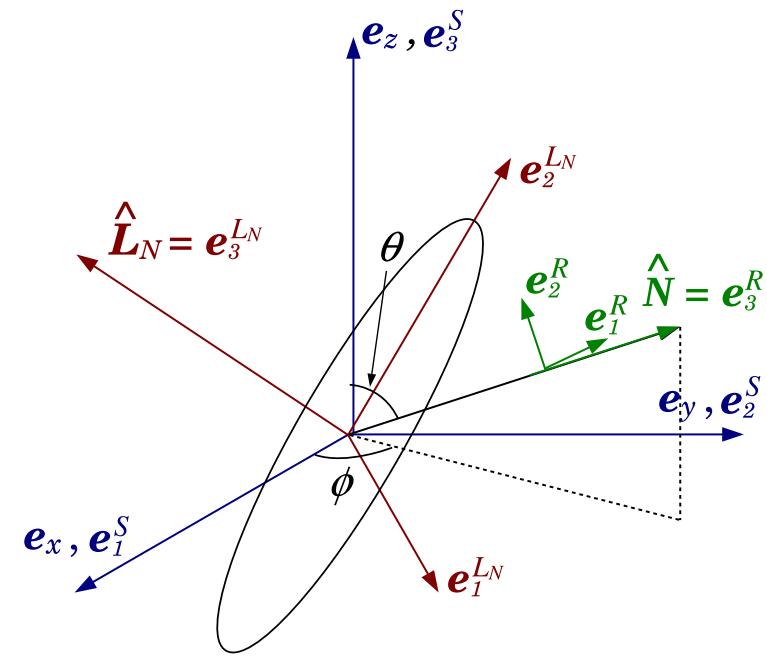

FIG. 1 (color online). We show the radiation frame $\left\{\boldsymbol{e}_{1}^{R}, \boldsymbol{e}_{2}^{R}, \boldsymbol{e}_{3}^{R}\right\}$, the inertial source frame $\left\{\boldsymbol{e}_{1}^{S}, \boldsymbol{e}_{2}^{S}, \boldsymbol{e}_{3}^{S}\right\}$, and the precessing source frame $\left\{\boldsymbol{e}_{1}^{L_{N}}, \boldsymbol{e}_{2}^{L_{N}}, \boldsymbol{e}_{3}^{L_{N}}\right\}$ employed to describe a precessing $\mathrm{BH}$ binary and its GW radiation.

basis vectors $\left\{\boldsymbol{e}_{1}^{S}, \boldsymbol{e}_{2}^{S}, \boldsymbol{e}_{3}^{S}\right\}$ for which the $\boldsymbol{e}_{3}^{S}$ is aligned with $\hat{\boldsymbol{L}}_{N}$. In the precessing case, we choose to align the source frame $\left\{\boldsymbol{e}_{1}^{S}, \boldsymbol{e}_{2}^{S}, \boldsymbol{e}_{3}^{S}\right\}$ with $\left\{\boldsymbol{e}_{x}, \boldsymbol{e}_{y}, \boldsymbol{e}_{z}\right\}$. The basis vector $\boldsymbol{e}_{3}^{S}$ is aligned with the initial orbital orientation $\hat{\boldsymbol{L}}_{N}(0)$ but not with $\hat{\boldsymbol{L}}_{N}(t)$ at later times.

The GW polarizations $h_{+}$and $h_{\times}$can be obtained by projecting the strain tensor $h_{i j}$ onto the radiation frame described by the (unit) basis vectors $\left\{\boldsymbol{e}_{1}^{R}, \boldsymbol{e}_{2}^{R}, \boldsymbol{e}_{3}^{R} \equiv \hat{\boldsymbol{N}}\right\}$, the basis vector $\hat{N}$ being along the direction of propagation of the wave (see Fig. 1). That is,

$$
\begin{aligned}
& h_{+}=\frac{1}{2}\left[\boldsymbol{e}_{1}^{R} \otimes \boldsymbol{e}_{1}^{R}-\boldsymbol{e}_{2}^{R} \otimes \boldsymbol{e}_{2}^{R}\right]^{i j} h_{i j}, \\
& h_{\times}=\frac{1}{2}\left[\boldsymbol{e}_{1}^{R} \otimes \boldsymbol{e}_{2}^{R}+\boldsymbol{e}_{2}^{R} \otimes \boldsymbol{e}_{1}^{R}\right]^{i j} h_{i j},
\end{aligned}
$$

where the basis vectors $\boldsymbol{e}_{1}^{R}$ and $\boldsymbol{e}_{2}^{R}$ are defined by (see Fig. 1)

$$
\begin{gathered}
\boldsymbol{e}_{1}^{R} \equiv \frac{\boldsymbol{e}_{z} \times \hat{\boldsymbol{N}}}{\left|\boldsymbol{e}_{z} \times \hat{\boldsymbol{N}}\right|}, \\
\boldsymbol{e}_{2}^{R} \equiv \hat{\boldsymbol{N}} \times \boldsymbol{e}_{1}^{R} .
\end{gathered}
$$

In the source frame $\left\{\boldsymbol{e}_{1}^{S}, \boldsymbol{e}_{2}^{S}, \boldsymbol{e}_{3}^{S}\right\}$, we can decompose the polarizations $h_{+}$and $h_{\times}$in -2 -spin-weighted spherical harmonics ${ }_{-2} Y_{\ell m}(\theta, \phi)$ as

$$
h_{+}(\theta, \phi)-i h_{\times}(\theta, \phi)=\sum_{\ell=2}^{\infty} \sum_{m=-\ell}^{\ell}{ }_{-2} Y_{\ell m}(\theta, \phi) h_{\ell m} .
$$

The modes $h_{\ell m}$ can be calculated by applying the orthogonality condition valid for the ${ }_{-2} Y_{\ell m}(\theta, \phi)$ 's. Thus,

$$
h_{\ell m}=\int\left[h_{+}(\theta, \phi)-i h_{\times}(\theta, \phi)\right]_{-2} Y_{\ell m}^{*}(\theta, \phi) d \Omega \text {, }
$$

where $\theta$ and $\phi$ are the inclination and azimuthal angles of the unit vector $\hat{N}$ as measured in the source frame. Note that in the above expressions, we omit the dependence of the GW polarizations on time and binary parameters.

\section{B. Precessing source frames in analytical-relativity waveforms}

In the nonprecessing case, the orbital orientation is constant and coincides with the directions of the orbital angular momentum $\boldsymbol{L} \equiv \mu M \boldsymbol{r} \times \boldsymbol{p}$, the Newtonian angular momentum $\boldsymbol{L}_{N} \equiv \mu M \boldsymbol{r} \times \dot{\boldsymbol{r}}$, and the total angular momentum $\boldsymbol{J} \equiv \boldsymbol{L}+\boldsymbol{S}_{1}+\boldsymbol{S}_{2}$. If the source frame is aligned with the orbital orientation, the gravitational polarizations are quite simple and are described mainly by the $(2,2)$ mode and a few subdominant modes $[12,13]$. In this case, the wave's amplitude and frequency increase monotonically during the inspiral and plunge stages, and the amplitudes of the GW modes display a clean hierarchy. By contrast, precessing waveforms decomposed in an inertial source frame show strong amplitude and phase modulations. In this case the amplitudes of the GW modes do not necessarily follow a clean hierarchy [47]. Ideally, we would like to conduct comparisons and calibrations between analytical and numerical waveforms in a time-dependent source frame that minimizes precession-induced modulations. Fortunately, this is possible if we choose a source frame that precesses with the binary orbital plane [25,26,29-31].

Buonanno, Chen, and Vallisneri [25] proposed the precessing convention that neatly disentangles precessional effects from both amplitude and phase evolutions in restricted (i.e., leading-order) PN waveforms. In the precessing convention, the precessing waveform is written as the product of a nonprecessing carrier waveform and a modulation term that collects all precessional effects. In Refs. $[25,26]$ the authors chose the precessing source frame aligned with the Newtonian orbital angular momentum $\boldsymbol{L}_{N}$. In this case, the basis vectors of the precessing source frame, $\left\{\boldsymbol{e}_{1}^{L_{N}}(t), \boldsymbol{e}_{2}^{L_{N}}(t), \boldsymbol{e}_{3}^{L_{N}}(t)\right\}$ in Fig. 1, read [25]

$$
\begin{gathered}
\boldsymbol{e}_{3}^{L_{N}}(t)=\hat{\boldsymbol{L}}_{N}(t), \\
\dot{\boldsymbol{e}}_{1}^{L_{N}}(t)=\Omega_{e}(t) \times \boldsymbol{e}_{1}^{L_{N}}(t), \\
\dot{\boldsymbol{e}}_{2}^{L_{N}}(t)=\Omega_{e}(t) \times \boldsymbol{e}_{2}^{L_{N}}(t),
\end{gathered}
$$

where

$$
\Omega_{e}(t) \equiv \Omega_{L}(t)-\left[\Omega_{L}(t) \cdot \hat{\boldsymbol{L}}_{N}(t)\right] \hat{\boldsymbol{L}}_{N}(t)=\hat{\boldsymbol{L}}_{N}(t) \times \dot{\hat{\boldsymbol{L}}}_{N}(t),
$$


and $\Omega_{L}(t)$ is the angular velocity of the precession of $\hat{\boldsymbol{L}}_{N}(t)$ and satisfies $\hat{\boldsymbol{L}}_{N}(t)=\Omega_{L}(t) \times \hat{\boldsymbol{L}}_{N}(t)$. Aligning the precessing source frame with $\hat{\boldsymbol{L}}_{N}(t)$ in Eq. (5a) removes the precession-induced amplitude modulations. Intuitively, Eqs. (5b)-(6) impose that $\boldsymbol{e}_{1}^{L_{N}}(t)$ and $\boldsymbol{e}_{2}^{L_{N}}(t)$ follow the precession of $\boldsymbol{e}_{3}^{L_{N}}(t)=\hat{\boldsymbol{L}}_{N}(t)$ but do not precess around it. The key point of the precessing convention is the removal of all precession-induced modulations from the orbital phase $\Phi(t)$ so that $\Phi(t)$ is simply given by the integral of the (monotonic) orbital frequency $\Omega$, i.e., $\Phi(t)=$ $\int \Omega\left(t^{\prime}\right) d t^{\prime}$ (see for details Sec. IV A in Ref. [25]). The freedom of choosing the constant of integration, or the initial phase, is degenerate with the only degree of freedom left in defining the precessing source frame, namely, a constant rotation of $\boldsymbol{e}_{1}^{L_{N}}$ and $\boldsymbol{e}_{2}^{L_{N}}$ around $\boldsymbol{e}_{3}^{L_{N}}$.

We want to test now the precessing convention on inspiraling PN waveforms computed beyond the restricted approximation, i.e., beyond leading order. We employ the waveforms of Ref. [47] that have spin-amplitude corrections through 1.5PN order. We decompose the $h_{\ell m}$ 's in two source frames: (i) the inertial source frame aligned with the initial total angular momentum $\boldsymbol{J}_{0}$ [47] and (ii) the precessing source frame $\left\{\boldsymbol{e}_{1}^{L_{N}}(t), \boldsymbol{e}_{2}^{L_{N}}(t), \boldsymbol{e}_{3}^{L_{N}}(t)\right\}$ defined by Eqs. (5a)-(6). The waveforms decomposed in the $\boldsymbol{J}_{0}$ frame are given explicitly in Appendix B of Ref. [47]. We calculate the waveforms decomposed in the precessing $\boldsymbol{L}_{N}(t)$ frame from the waveforms decomposed in the $\boldsymbol{J}_{0}$ frame by properly rotating the $h_{\ell m}$ modes.

In general, given a set of spin-weighted spherical harmonic $h_{\ell m}^{(o)}$ decomposed in an original frame and the Euler angles $(\alpha, \beta, \gamma)$ that define the rotation from the original frame to a new frame, the modes $h_{\ell m}^{(n)}$ decomposed in the new frame are given by $[47,48]$

$$
h_{\ell m}^{(n)}=\sum_{m^{\prime}=-\ell}^{\ell} D_{m^{\prime} m}^{\ell *}(\alpha, \beta, \gamma) h_{\ell m^{\prime}}^{(o)},
$$

where $D_{m^{\prime} m}^{\ell *}(\alpha, \beta, \gamma)$ is the complex conjugate of the Wigner $D$ matrix

$$
D_{m^{\prime} m}^{\ell}(\alpha, \beta, \gamma)=(-1)^{m^{\prime}} \sqrt{\frac{4 \pi}{2 \ell+1}-m^{\prime}} Y_{\ell m}(\beta, \alpha) e^{i m^{\prime} \gamma},
$$

where ${ }_{-m^{\prime}} Y_{\ell m}$ is the spherical harmonic of spin-weight $-m^{\prime}$. The transformation is closed among modes with the same index $\ell$. In this paper, we focus on the $\ell=2$ modes both for simplicity and because even when precession is present the $\ell=2$ modes still dominate. Nevertheless, the $\ell>2$ modes are not negligible, and we plan to extend the precessing EOB model to those modes in the future, following the same approach we propose and demonstrate here with the $\ell=2$ modes.

In Fig. 2 we compare the $\boldsymbol{J}_{0}$-frame and $\boldsymbol{L}_{N}(t)$-frame $h_{2 m}$ inspiraling waveforms emitted by a BH binary with mass

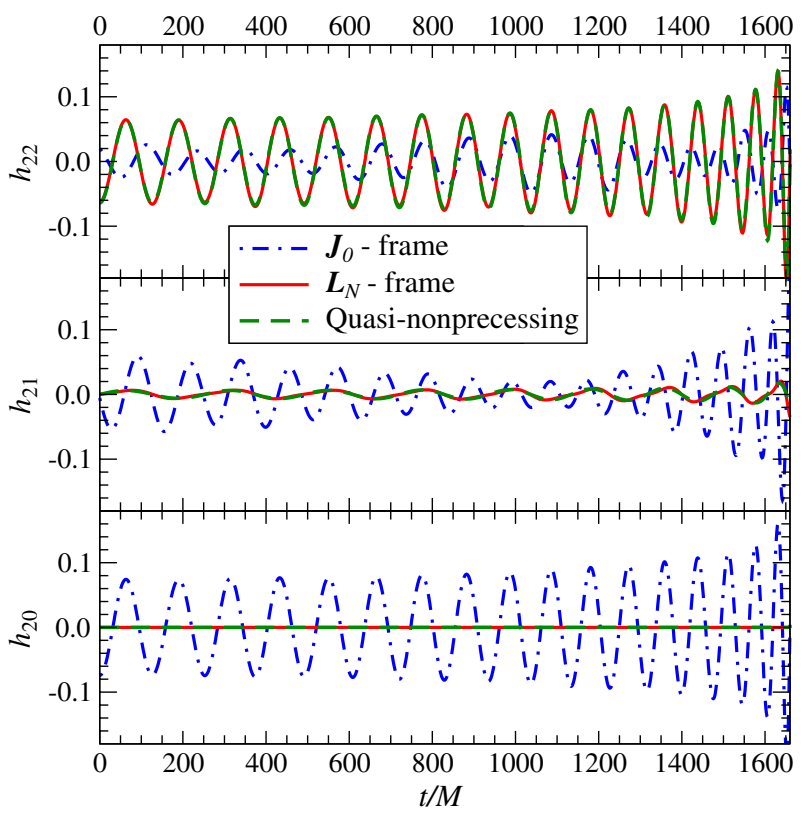

FIG. 2 (color online). We show inspiraling, precessing PN waveforms decomposed in the inertial source frame aligned with the initial total angular momentum $\boldsymbol{J}_{0}$ and in the precessing source frame aligned with the Newtonian orbital angular momentum $\boldsymbol{L}_{N}(t)$. For comparison, we show also the quasinonprecessing PN waveforms defined in Sec. II B. They lie on top of the $\boldsymbol{L}_{N}(t)$-frame waveforms. The three panels use the same scale on the $y$ axis so that the amplitudes of the modes $h_{22}, h_{21}$, and $h_{20}$ can be easily compared.

ratio $q=6$ and spin magnitudes $\chi_{1}=\chi_{2}=0.8$. We choose spin orientations that give strong precession-induced modulations. As we can see, there is a clear hierarchy among the $h_{2 m}$ 's amplitudes when decomposed in the $\boldsymbol{L}_{N}(t)$ frame but not when the decomposition is done in the $\boldsymbol{J}_{0}$ frame. In fact, in the $\boldsymbol{J}_{0}$ frame, the $(2,1)$ and $(2,0)$ modes have even larger amplitudes than the $(2,2)$ mode. We notice that the strong amplitude and phase modulations of the modes in the $\boldsymbol{J}_{0}$ frame almost completely disappear when the $\boldsymbol{L}_{N}(t)$ frame is used.

Furthermore, we find it interesting to compare the modes of the precessing waveforms to the ones of a "nonprecessing" binary system having the same mass ratio and $\chi_{i}(t) \equiv \chi_{i} \hat{\boldsymbol{S}}_{i}(t) \cdot \hat{\boldsymbol{L}}_{N}(t),(i=1,2)$. That is to say, we keep only the components of the spin vectors along the direction of the Newtonian angular momentum $\boldsymbol{L}_{N}(t)$ and set all the other components to zero. For convenience, we refer to such nonprecessing waveforms as the quasinonprecessing waveforms. We use the adjective "quasi" because, differently from the nonprecessing waveforms, where the spins are aligned or antialigned with the orbital angular momentum and remain constant throughout the evolution, in the quasinonprecessing waveforms, the spins evolve [according to Eqs. (11c)-(11d) given below] and over time change their projections onto $\boldsymbol{L}_{N}$. As can be seen in Fig. 2, the near-perfect agreement between $\boldsymbol{L}_{N}(t)$-frame and 
quasinonprecessing waveforms indicates that the spin components along $\boldsymbol{L}_{N}(t)$ dominate the spin effects. This conjecture is reinforced by the observation that, because of parity symmetry, the spin-orbit couplings contribute to the GW energy flux to infinity (known through 3.5PN order) only through terms of the form $\boldsymbol{S}_{i}(t) \cdot \hat{\boldsymbol{L}}_{N}(t),(i=1,2)$ $[20,21,49,50]$. The energy flux is a frame independent quantity. It is given, in the adiabatic assumption, by ${ }^{2}$

$$
\frac{d E}{d t}=\frac{(M \Omega)^{2}}{8 \pi} \sum_{\ell=2}^{\infty} \sum_{m=-\ell}^{\ell} m^{2}\left|\frac{\mathcal{R}}{M} h_{\ell m}\right|^{2},
$$

where $\mathcal{R}$ is the distance to the source (and simply cancels the dependence on $\mathcal{R}$ hidden in the $h_{\ell m}$ 's). The fact that the spin-orbit effects in $d E / d t$ depend on spins only through $\boldsymbol{S}_{i}(t) \cdot \hat{\boldsymbol{L}}_{N}(t),(i=1,2)$ suggests that the dependence of the modes on spin's components parallel to the orbital plane disappears when all the modes are summed up to make the total energy flux.

Therefore, beyond the leading-order results of Ref. [25], we find that PN precessing waveforms [47] reduce to nearly nonprecessing waveforms when decomposed to spinweighted spherical harmonics in the source frame $\left\{\boldsymbol{e}_{1}^{L_{N}}(t), \boldsymbol{e}_{2}^{L_{N}}(t), \boldsymbol{e}_{3}^{L_{N}}(t)\right\}$ of the precessing convention [25]. In addition, the PN quasinonprecessing waveforms are good first approximations to the $h_{\ell m}$ 's modes in the precessing frame.

\section{Precessing source frames in numerical-relativity waveforms}

The possibility of demodulating precessing waveforms using precessing source frames was recently verified with and generalized to NR waveforms in Refs. [27,29,30]. In particular, Schmidt et al. [27,29] and O'Shaughnessy et al. [30] identified the preferred radiation axis at infinity and showed that if a precessing frame aligned with the radiation axis is chosen then the amplitude and phase modulations of numerical waveforms are removed and a clean hierarchy among the modes is restored.

In particular, Schmidt et al. proposed the so-called quadrupole-preferred frame in which the power of the $(\ell, \pm m)=(2,2)$ mode is maximized. O'Shaughnessy et al. proposed a more general and geometrical choice of the precessing frame in which the $z$ component of the radiated angular momentum is maximized. The latter proposal reduces to the choice of Schmidt et al. when the radiated angular momentum is calculated using only the $(\ell, \pm m)=$ $(2,2)$ modes. Boyle et al. [31] then proposed the minimal rotation condition to remove the remaining arbitrariness in

\footnotetext{
${ }^{2}$ Following the nonprecessing EOB model of Ref. [13], we include in $d E / d t$ the spin-orbit terms through $1.5 \mathrm{PN}$ order [51], even if some of us have recently computed the spin-orbit and spin-spin terms in the factorized flux through 2PN order using results in Ref. [22].
}

the azimuthal rotation of the precessing frame and in the phase modulations of the waveform. Given an inertial frame $\left\{\boldsymbol{e}_{x}, \boldsymbol{e}_{y}, \boldsymbol{e}_{z}\right\}$ and the first two Euler angles $\alpha(t)$ and $\beta(t)$ that align $\boldsymbol{e}_{z}$ with the radiation axis, the minimal rotation condition on the third Euler angle $\gamma(t)$ is given by

$$
\dot{\gamma}(t)=-\dot{\alpha}(t) \cos \beta(t) .
$$

This condition is equivalent to Eqs. (5b)-(6) above on the evolution of $\boldsymbol{e}_{1}^{L_{N}}(t)$ and $\boldsymbol{e}_{2}^{L_{N}}(t)$. If $\alpha(t)$ and $\beta(t)$ are the first two Euler angles that align $\boldsymbol{e}_{z}$ with $\hat{\boldsymbol{L}}_{N}$, then $\gamma(t)-\gamma\left(t_{0}\right)$ is the angle by which $\boldsymbol{e}_{1}^{L_{N}}(t)$ and $\boldsymbol{e}_{2}^{L_{N}}(t)$ shall rotate in the instantaneous orbital plane, relative to their positions at a reference time $t_{0}$, to satisfy the precession convention. Recently, Boyle [32] proposed a geometric definition of the angular-velocity vector of a waveform, to determine a frame in which the modes' amplitudes become very simple and the phases are nearly constant.

Schmidt et al. [27] showed that precessing PN inspiral waveforms computed in the precessing source frame aligned with the preferred radiation axis are well approximated by nonprecessing PN waveforms. Furthermore, they proposed that precessing waveforms can be generated with good accuracy by transforming nonprecessing waveforms from precessing source frames to inertial source frames. In a recent study, Pekowsky et al. [33] studied the mapping of precessing waveforms to nonprecessing ones using a large number of (short) numerical simulations and the analytical IMRPhenomB [17] waveforms. They found that precessional degrees of freedom that cannot be reproduced by nonprecessing models (such as spin's components perpendicular to $\hat{\boldsymbol{L}}_{N}$ ) give rise to corrections to the nonprecessing waveforms that are very small during inspiral, but they can become significant during merger and ringdown.

\section{Strategy to build precessing effective-one-body waveforms}

Motivated by the results discussed in Secs. II B and II C of a nearly complete separation of precession-induced modulations in precessing waveforms when using appropriate precessing source frames, we propose the following approach to generate generic EOB waveforms.

First, we evolve the EOB dynamics and solve Eqs. (5a)-(6) for the precessing source frame $\left\{\boldsymbol{e}_{1}^{L_{N}}(t)\right.$, $\left.\boldsymbol{e}_{2}^{L_{N}}(t), \boldsymbol{e}_{3}^{L_{N}}(t)=\hat{\boldsymbol{L}}_{N}(t)\right\}$. Since the difference between $\boldsymbol{L}_{N}$ and $\boldsymbol{L}$ starts at $1 \mathrm{PN}$ order, the leading-order conclusions achieved by the precessing $\boldsymbol{L}_{N}$ frame hold if we replace $\boldsymbol{L}_{N}$ with $\boldsymbol{L}$ in Eqs. (5a)-(6). We have verified that precessing waveforms decomposed in the $\boldsymbol{L}$ frame agree equally well with the quasinonprecessing waveforms generated by keeping only spin's components along $\boldsymbol{L}$. Furthermore, in Sec. IV we compare precessing EOB waveforms (generated either in the $\boldsymbol{L}_{N}$ frame or in the $\boldsymbol{L}$ frame) to $\mathrm{NR}$ waveforms and find that their mutual difference is 
marginal. Without a more accurate calibration and comprehensive comparisons with NR waveforms, we do not know a priori whether the $\boldsymbol{L}_{N}$ frame or the $\boldsymbol{L}$ frame is more preferable, nor can we say which of them captures the precession effects more faithfully. Thus, at the current stage, we simply adopt the $\boldsymbol{L}_{N}$-frame as the default precessing source frame in the EOB model.

Second, because of the simple features of the inspiralplunge modes in the precessing source frame-little modulation and clean hierarchy-we choose to model the precessing inspiral-plunge EOB modes in this frame and generate modes in any arbitrary source frame through Eq. (7). Since factorized EOB modes for precessing spins are not available yet and EOB modes have been calibrated only to nonspinning and spinning, nonprecessing NR modes $[12,13]$, we choose to work in the precessing source frame and use quasinonprecessing modes as good approximations to precessing modes (as discussed in Secs. II B and II C). In particular, we employ the quasinonprecessing inspiral-plunge modes based on the latest spinning, nonprecessing EOB model that was calibrated to NR modes in Ref. [13]. Note that we are not obliged to use in the future quasinonprecessing waveforms in the precessing source frame. In fact, using the same EOB dynamics for the comparable-mass binary configurations considered in this paper, we find that the Taylor-expanded precessing 2.5PN modes [47] generated in the inertial frame are practically indistinguishable from the EOB modes generated through the frame-rotation procedures described above. However, unlike the factorized resummed modes, Taylor-expanded PN modes agree much worse with Teukolsky waveforms in the test-particle limit [51], especially for large spins [52]. We thus expect the precessing model based on factorizedresummed quasinonprecessing waveforms to be more reliable when extrapolated beyond the comparable-mass configurations. As soon as factorized EOB modes for precessing spins become available, we shall relax the assumption of using quasinonprecessing inspiral-plunge modes $^{3}$. The strategy that we present in this paper is generic and can easily be applied to future calibrations and analytical improvements of the EOB model.

Third, we rotate the quasinonprecessing modes from the precessing source frame to the inertial frame for which the $z$ axis coincides with the direction of the total angular momentum $\boldsymbol{J}$ at a time very close to merger when the direction of $\boldsymbol{J}$ is a good approximation to the direction of the spin of the final $\mathrm{BH}$. In this inertial frame, we match the inspiral-plunge to merger-ringdown modes following the usual prescription in the EOB approach [13]. After generating inspiral-merger-ringdown modes in this frame,

\footnotetext{
${ }^{3}$ It remains to be investigated, though, whether it is necessary to include precessing effects in the EOB modes decomposed in the precessing source frame to meet more stringent accuracy requirements for advanced LIGO and Virgo searches.
}

it is straightforward to calculate $\mathrm{EOB}$ modes $h_{\ell m}$ in any source frame or EOB polarizations $h_{+, x}$ in any radiation frame.

\section{PRECESSING EFFECTIVE-ONE-BODY MODEL}

In this section we construct a generic, precessing EOB model following the general strategy outlined above-it employs the precessing source frame introduced in Ref. [25] and the quasinonprecessing waveforms based on the nonprecessing EOB model developed in Ref. [13].

\section{A. Effective-one-body dynamics}

Since we employ exactly the same EOB dynamics calibrated against NR simulations in Ref. [13], we review only the key ingredients of the dynamics and refer the readers to Ref. [13] for further details.

The EOB dynamics of spinning BH binary systems is obtained solving the Hamilton equations

$$
\begin{gathered}
\frac{d \boldsymbol{r}}{d \hat{t}}=\left\{\boldsymbol{r}, \hat{H}_{\text {real }}\right\}=\frac{\partial \hat{H}_{\text {real }}}{\partial \boldsymbol{p}}, \\
\frac{d \boldsymbol{p}}{d \hat{t}}=\left\{\boldsymbol{p}, \hat{H}_{\text {real }}\right\}+\hat{\mathcal{F}}=-\frac{\partial \hat{H}_{\text {real }}}{\partial \boldsymbol{r}}+\hat{\mathcal{F}}, \\
\frac{d \boldsymbol{S}_{1}}{d t}=\left\{\boldsymbol{S}_{1}, \mu \hat{H}_{\text {real }}\right\}=\mu \frac{\partial \hat{H}_{\text {real }}}{\partial \boldsymbol{S}_{1}} \times \boldsymbol{S}_{1}, \\
\frac{d \boldsymbol{S}_{2}}{d t}=\left\{\boldsymbol{S}_{2}, \mu \hat{H}_{\text {real }}\right\}=\mu \frac{\partial \hat{H}_{\text {real }}}{\partial \boldsymbol{S}_{2}} \times \boldsymbol{S}_{2},
\end{gathered}
$$

where $\hat{t} \equiv t / M$ is the dimensionless time variable, $\hat{H}_{\text {real }}$ is the reduced EOB Hamiltonian derived in Refs. [39-41] and reviewed in Sec. II A of Ref. [13], and $\hat{\mathcal{F}}$ is the reduced radiation reaction force. Following Ref. [38], we use

$$
\hat{\mathcal{F}}=\frac{1}{\nu \hat{\Omega}|\boldsymbol{r} \times \boldsymbol{p}|} \frac{d E}{d t} \boldsymbol{p},
$$

where $\hat{\Omega} \equiv M|\boldsymbol{r} \times \dot{\boldsymbol{r}}| / r^{2}$ is the dimensionless orbital frequency and $d E / d t$ is the energy flux for quasispherical orbits. We use Eq. (9) with $\ell \leq 8$, namely,

$$
\frac{d E}{d t}=\frac{\hat{\Omega}^{2}}{8 \pi} \sum_{\ell=2}^{8} \sum_{m=-\ell}^{\ell} m^{2}\left|\frac{\mathcal{R}}{M} h_{\ell m}\right|^{2} .
$$

Because under a change of frame the $h_{\ell m}$ modes for a given $\ell$ transform into modes with the same $\ell$, Eq. (13) is still frame independent. We insert the quasinonprecessing modes $h_{\ell m}$, i.e., the modes decomposed in the precessing source frame aligned with $\boldsymbol{L}_{N}(t)$, into Eq. (13). The quasinonprecessing modes can be calculated directly in 
the inertial frame $\left\{\boldsymbol{e}_{x}, \boldsymbol{e}_{y}, \boldsymbol{e}_{z}\right\}$ in which we solve the EOB dynamics. The only difference from the procedure of Ref. [13] is to replace the constant $\chi_{1}$ and $\chi_{2}$ with $\chi_{1} \hat{S}_{1}(t)$. $\hat{\boldsymbol{L}}_{N}(t)$ and $\chi_{2} \hat{\boldsymbol{S}}_{2}(t) \cdot \hat{\boldsymbol{L}}_{N}(t)$.

\section{B. Initial conditions}

For applications in data analysis and comparisons with numerical or analytical waveforms, we need initial conditions that start the orbital evolution with sufficiently small eccentricity at a given orbital separation (or GW frequency) and spins orientation. The analytical quasispherical initial conditions proposed in Ref. [38] are a good first approximation.

In the nonprecessing case [11-13], the residual eccentricity can be further reduced by starting the evolution at a larger separation (smaller GW frequency) and waiting for the orbits to be better circularized by the radiation reaction. In the precessing case, however, we cannot easily reduce the eccentricity in this way because we need specific spin directions at the initial separation. To reduce eccentricity by starting the evolution at a larger separation, we need to figure out what are the spin directions at this larger separation to ensure the desired spin directions at a given (smaller) initial separation. To reach this goal and reduce the eccentricity for quasispherical initial conditions, we employ the method developed in Ref. [53], which is based on Refs. $[43,54]^{4}$. Thus, we first evolve the binary for a few orbits and estimate the eccentricity through oscillations in orbital frequency $\Omega$ and separation $r$. We then apply corrections to the initial conditions following Eqs. (74) and (75) in Ref. [53]. We repeat these steps until the eccentricity is sufficiently small.

\section{Nonprecessing effective-one-body waveforms}

The EOB nonprecessing inspiral-plunge modes $h_{\ell m}^{\mathrm{NP} \text {, insp-plunge }}$ developed in Ref. [13] are given by

$$
h_{\ell m}^{\mathrm{NP}, \text { insp-plunge }}=h_{\ell m}^{\mathrm{F}} N_{\ell m},
$$

where $h_{\ell m}^{\mathrm{F}}$ are the factorized modes derived in Refs. [51,59,60] and $N_{\ell m}$ are nonquasicircular (NQC) corrections that model deviations from the quasicircular motion, which is assumed when deriving $h_{\ell m}^{\mathrm{F}}$. The factorized modes read

$$
h_{\ell m}^{\mathrm{F}}=h_{\ell m}^{(N, \epsilon)} \hat{S}_{\mathrm{eff}}^{(e)} T_{\ell m} e^{i \delta_{\ell m}}\left(\rho_{\ell m}\right)^{\ell},
$$

where $\epsilon$ is the parity of the mode. All the factors entering $h_{\ell m}^{\mathrm{F}}$ can be explicitly found in the Appendix of Ref. [13]. As discussed above, when using these expressions to generate quasinonprecessing modes, the only minor

\footnotetext{
${ }^{4}$ This method has been employed to reduce eccentricity in NR simulations of BH binary systems [44,45,55-58].
}

modification we have to take into account is to replace the constant spin magnitudes $\chi_{1}$ and $\chi_{2}$ by their timedependent counterparts. More specifically, in the nonprecessing case, the leading-order spin-orbit effects in $\rho_{\ell m}$ are parametrized by two linear combinations of the constant dimensionless spin parameters:

$$
\begin{aligned}
& \chi_{S} \equiv \frac{\chi_{1}+\chi_{2}}{2}, \\
& \chi_{A} \equiv \frac{\chi_{1}-\chi_{2}}{2} .
\end{aligned}
$$

In the precessing case, both $\chi_{S}$ and $\chi_{A}$ become linear combinations of the time-varying spin vectors projected along $\hat{\boldsymbol{L}}_{N}(t)$,

$$
\begin{aligned}
& \chi_{S}(t) \equiv \frac{1}{2}\left(\frac{\boldsymbol{S}_{1}(t)}{m_{1}^{2}}+\frac{\boldsymbol{S}_{2}(t)}{m_{2}^{2}}\right) \cdot \hat{\boldsymbol{L}}_{N}(t), \\
& \chi_{A}(t) \equiv \frac{1}{2}\left(\frac{\boldsymbol{S}_{1}(t)}{m_{1}^{2}}-\frac{\boldsymbol{S}_{2}(t)}{m_{2}^{2}}\right) \cdot \hat{\boldsymbol{L}}_{N}(t) .
\end{aligned}
$$

In Ref. [13] the inspiral-merger-ringdown mode $(2,2)$ was calibrated against NR simulations. Studies in the testparticle nonspinning [60] and spinning, nonprecessing [51] cases suggest that the factorized modes $h_{\ell m}^{\mathrm{F}}$ are good approximations of the inspiral-plunge modes even without any NQC correction or calibration. Thus, we model the inspiral-plunge $(2,1)$ mode with $h_{21}^{\mathrm{F}}$. The $(2,0)$ mode has been computed in PN theory at leading order, and its amplitude is $5 / 14 \sqrt{6} \simeq 0.15$ times the amplitude of the leading-order $(2,2)$ mode [61]. However, this prediction does not agree with NR results. In fact, we find [62] that for the nonspinning NR simulations of mass ratios $q=1,6$ [12], the amplitude of the $(2,0)$ mode during the inspiral is smaller than the one of the $(2,2)$ mode by a factor $\sim 10^{3}$. Since we do not yet understand the origin of this discrepancy, we have decided that in this first investigation we neglect the nonprecessing EOB $(2,0)$ mode.

The NQC correction to the $(2,2)$ mode, $N_{22}$, is given by

$$
\begin{aligned}
N_{22}= & {\left[1+\left(\frac{p_{r^{*}}}{r \hat{\Omega}}\right)^{2}\left(a_{1}^{h_{22}}+\frac{a_{2}^{h_{22}}}{r}+\frac{a_{3}^{h_{22}}}{r^{3 / 2}}+\frac{a_{4}^{h_{22}}}{r^{2}}+\frac{a_{5}^{h_{22}}}{r^{5 / 2}}\right)\right] } \\
& \times \exp \left[i \frac{p_{r^{*}}}{r \hat{\Omega}}\left(b_{1}^{h_{22}}+p_{r^{*}}^{2} b_{2}^{h_{22}}+\frac{p_{r^{*}}^{2}}{r^{1 / 2}} b_{3}^{h_{22}}+\frac{p_{r^{*}}^{2}}{r} b_{4}^{h_{22}}\right)\right],
\end{aligned}
$$

where the amplitude coefficients $a_{i}^{h_{22}}$ (with $i=1 \ldots 5$ ) and the phase coefficients $b_{i}^{h_{22}}$ (with $i=1 \ldots 4$ ) are obtained through the iterative procedure described in Sec. II B of Ref. [13]. Since only equal-mass, equal-spin, nonprecessing NR simulations were used to calibrate the EOB model of Ref. [13], we have to map the $N_{22}$ from generic spin configurations to equal-spin, nonprecessing configurations. 
Without further calibrations, we first adopt a mapping from precessing to nonprecessing configurations that equates the $\chi_{S}(0)$ and $\chi_{A}(0)$ of a precessing configuration [defined in Eqs. (17a) and (17b)] to the constant $\chi_{S}$ and $\chi_{A}$ of a nonprecessing configuration. Then, we apply the mapping from a generic nonprecessing configuration to an equalspin, nonprecessing configurations as defined in Sec. IV A of Ref. [13].

\section{Precessing source frame}

Let $\boldsymbol{e}_{3}^{L_{N}}(t)=\hat{\boldsymbol{L}}_{N}(t)$ be the third (unit) basis vector of the precessing source frame. We solve the other two (unit) basis vectors $\boldsymbol{e}_{1}^{L_{N}}(t)$ and $\boldsymbol{e}_{2}^{L_{N}}(t)$ by applying the minimal rotation condition. We do it because the latter involves only one differential equation, namely, Eq. (10), instead of Eqs. (5b) and (5c) for the precessing convention. Specifically, with the help of the inertial source frame $\left\{\boldsymbol{e}_{x}^{S}, \boldsymbol{e}_{y}^{S}, \boldsymbol{e}_{z}^{S}\right\}$, we define

$$
\begin{aligned}
& \alpha(t)=\arctan \left[\frac{\boldsymbol{e}_{3}^{L_{N}}(t) \cdot \boldsymbol{e}_{y}^{S}}{\boldsymbol{e}_{3}^{L_{N}}(t) \cdot \boldsymbol{e}_{x}^{S}}\right], \\
& \beta(t)=\arccos \left[\boldsymbol{e}_{3}^{L_{N}}(t) \cdot \boldsymbol{e}_{z}^{S}\right],
\end{aligned}
$$

and solve ${ }^{5}$

$$
\dot{\gamma}(t)=-\dot{\alpha}(t) \cos \beta(t) .
$$

Those Euler angles $\alpha(t), \beta(t)$, and $\gamma(t)$ describe the time-dependent rotation from the inertial source frame $\left\{\boldsymbol{e}_{x}^{S}, \boldsymbol{e}_{y}^{S}, \boldsymbol{e}_{z}^{S}\right\}$ to the precessing source frame $\left\{\boldsymbol{e}_{1}^{L_{N}}(t), \boldsymbol{e}_{2}^{L_{N}}(t), \boldsymbol{e}_{3}^{L_{N}}(t)\right\}$ with the latter satisfying the minimal rotation condition. The only freedom in the definition of the precessing source frame is a constant shift in $\gamma(t)$ that is degenerate with the initial orbital phase.

\section{E. Precessing effective-one-body waveforms}

We build the complete inspiral-plunge-merger-ringdown waveforms in an inertial frame following the usual procedure in the EOB approach [6-14,34,35,63]. More specifically, we join the inspiral-plunge waveform $h_{\ell m}^{\text {insp-plunge }}$ and the merger-ringdown waveform $h_{\ell m}^{\text {merger-RD }}$ at a matching time $t_{\text {match }}^{\ell m}$ as

$$
\begin{aligned}
h_{\ell m}^{\mathrm{EOB}}(t)= & h_{\ell m}^{\text {inspiral-plunge }}(t) \theta\left(t_{\text {match }}^{\ell m}-t\right) \\
& +h_{\ell m}^{\text {merger-RD }}(t) \theta\left(t-t_{\text {match }}^{\ell m}\right) .
\end{aligned}
$$

\footnotetext{
${ }^{5}$ Following Boyle et al. [31], we integrate $\gamma(t)$ by parts and implement $\quad \gamma(t)=-\alpha(t) \cos \beta(t)-\int \alpha(t) \dot{\beta}(t) \sin \beta(t) d t \quad$ to avoid differentiating $\alpha(t)$, which can be noisy near the coordinate singularities at $\beta(t)=0$ and $\beta(t)=\pi$. We note that Boyle [32] recently proposed a much more accurate and robust method to integrate $\gamma(t)$ using quaternions.
}

Given the quasinonprecessing inspiral-plunge modes

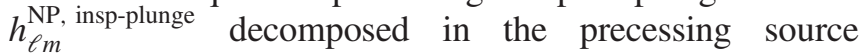
frame and the Euler angles [not necessarily those in Eqs. (19)-(20), which are specific to $\left.\left\{\boldsymbol{e}_{x}^{S}, \boldsymbol{e}_{y}^{S}, \boldsymbol{e}_{z}^{S}\right\}\right]$ defining the rotation from the precessing source frame to any inertial frame, the inspiral-plunge modes in the inertial frame are given by Eqs. (7). To study the $h_{2 m}$ modes, we need all $\ell=2$ modes in the precessing source frame. As discussed in Sec. III C, we employ the calibrated $(2,2)$ mode of Ref. [13], $h_{21}^{\mathrm{F}}$ for the $(2,1)$ mode and zero for the $(2,0)$ mode. In the precessing source frame, since we use quasinonprecessing inspiral-plunge modes to approximate precessing modes, we further assume reflection symmetry, which, combined with parity invariance, gives modes with $m<0$ through $h_{2,-m}^{\mathrm{NP} \text { insp-plunge }}(t)=h_{2 m}^{\mathrm{NP} \text {, insp-plunge }}(t)$. Pekowsky et al. [33] discussed how this symmetry is broken by precessional effects, giving rise to a contribution to the $(2,2)$ mode which is odd under reflection. In the only example investigated in Ref. [33], the ratio between the component of the $(2,2)$ mode of the Weyl scalar $\Psi_{4,22}$ that is odd under reflection and the one that is even under reflection is $\sim 0.01$, while the ratio between the former and the $(2,1)$ mode of the Weyl scalar $\Psi_{4,21}$ is $\sim 1$. Since the Weyl scalar and the metric perturbation are related by $\Psi_{4, \ell m}=\ddot{h}_{\ell m} \simeq m^{2} \hat{\Omega}^{2} h_{\ell m}$ during the inspiral, the odd component of the $h_{22}$ 's amplitude is about a fourth of the $h_{21}$ 's. The odd component of the $h_{22}$ 's amplitude becomes substantial only during the merger and ringdown. Thus, in this first study, we ignore the component of the $(2,2)$ mode that is odd under reflection when describing the inspiraling waveform in the precessing frame, but we include the odd component when building the merger-ringdown waveform.

It is convenient to choose an inertial frame in which the merger-ringdown waveforms take simple forms. A natural choice is the frame aligned with the spin of the final $\mathrm{BH}$, in which the merger-ringdown waveforms are expressed as linear combinations of the quasinormal modes (QNMs) [6-14,34,35,63]. Barausse et al. [64] found strong evidence that the spin of the final $\mathrm{BH}$ is aligned with the initial total angular momentum of the binary. Using this assumption they derived accurate formulas for the final spin of a $\mathrm{BH}$ formed by merger. The success of their model verifies the PN-motivated assumption that the radiated angular momentum averaged over precessional cycles is almost aligned with the total angular momentum. Thus, the direction of the latter is preserved with high accuracy during the inspiral [23]. Here we employ the formulas in Ref. [64] to predict the magnitude of the spin of the final $\mathrm{BH}$, and we align the final-spin direction with $\boldsymbol{J}\left(t_{\Omega \text { peak }}^{\mathrm{EOB}}\right)$, which is the total angular momentum at the time the EOB orbital frequency reaches its peak $\left(t_{\Omega \text { peak }}^{\mathrm{EOB}}\right)$. The time $t_{\Omega \text { peak }}^{\mathrm{EOB}}$ has been adopted in most previous EOB models as the reference time of merger [9-14]. Without further information from NR simulations of precessing, spinning $\mathrm{BHs}$, we consider $\boldsymbol{J}\left(t_{\Omega \text { peak }}^{\mathrm{EOB}}\right)$ our best prediction of the final-spin direction. 
We expect that not a lot of angular momentum is radiated during the swift transition from merger to ringdown [63], and the small amount being radiated is likely to be nearly aligned with $\boldsymbol{J}\left(t_{\Omega \text { peak }}^{\mathrm{EOB}}\right)$. Besides, in the rare case of transitional precession, the assumption of Ref. [64] does not hold anymore, while our choice of $\boldsymbol{J}\left(t_{\Omega \text { peak }}^{\mathrm{EOB}}\right)$ is still valid.

The inspiral-plunge waveform $h_{\ell m}^{\text {insp-plunge }}$ in the inertial frame aligned with $\boldsymbol{J}\left(t_{\Omega \text { peak }}^{\mathrm{EOB}}\right)$ contains NQC corrections from the nonprecessing $(2,2)$ mode $h_{\ell m}^{\mathrm{NP} \text {, insp-plunge }}$. Those corrections are derived based on the assumption that the inspiralplunge waveforms in the precessing frame are the calibrated nonprecessing waveforms generated with the specific mapping of spin parameters defined in Sec. III C. Although we expect that such an assumption introduces systematic errors in $h_{\ell m}^{\text {insp-plunge }}$, we are not able to quantify them before comparing $h_{\ell m}^{\text {insp-plunge }}$ with precessing NR waveforms. Therefore, we do not apply any further correction to the inspiral-plunge waveform in this model. This choice also guarantees that $h_{\ell m}^{\text {insp-plunge }}$ modes reduce to the calibrated modes of Ref. [13] in the nonprecessing limit.

The merger-ringdown waveform is built following almost exactly the approach described in Ref. [13]. We first give a brief review of this approach and then describe the differences. The merger-ringdown waveform is modeled by a linear superposition of the QNMs of the final Kerr $\mathrm{BH}$ as

$$
h_{\ell m}^{\text {merger-RD }}(t)=\sum_{n=0}^{N-1} A_{\ell m n} e^{-i \sigma_{\ell m n}\left(t-t_{\text {match }}^{\ell m}\right)},
$$

where $N$ is the number of overtones, $A_{\ell m n}$ is the complex amplitude of the $n$th overtone of the $(\ell, m)$ mode, and $\sigma_{\ell m n}$ is the complex frequency of the $n$th overtone. The complex frequencies are known functions of the mass and spin of the final $\mathrm{BH}$ [65]. The mass of the final $\mathrm{BH}$ is given in Eq. (8) of Ref. [66]. The spin magnitude of the final $\mathrm{BH}$, as discussed earlier, is given in Eqs. (6), (8), and (10) of Ref. [64]. Following Ref. [13], we replace the highest physical overtone (the 7th) of the $(2,2)$ mode with a pseudoQNM for which a calibrated complex frequency is given in Eqs. (35a) and (35b) of Ref. [13]. Finally, we fix the complex amplitudes $A_{\ell m n}$ though a matching procedure [12] that imposes a $C^{1}$-smooth connection over a time interval $\Delta t_{\text {match }}^{\ell m}$ between the merger-ringdown waveform and the inspiral-plunge waveform, in the inertial frame aligned with $\boldsymbol{J}\left(t_{\Omega \text { peak }}^{\mathrm{EOB}}\right)$.

In Ref. [13] the matching time $t_{\text {match }}^{\ell m}$ and the time interval $\Delta t_{\text {match }}^{\ell m}$ were calibrated only for the $(2,2)$ mode. Here we need to specify those quantities also for the remaining $\ell=$ 2 modes. We find that in order to keep the matching procedure stable when the binary is strongly precessing around merger, we have to introduce in $t_{\text {match }}^{\ell m}$ and $\Delta t_{\text {match }}^{\ell m}$ a dependence on how much the orbital and total angular momentum are misaligned at merger, i.e., on the quantity $\hat{\boldsymbol{L}}\left(t_{\Omega \text { peak }}^{\mathrm{EOB}}\right) \cdot \hat{\boldsymbol{J}}\left(t_{\Omega \text { peak }}^{\mathrm{EOB}}\right)$. More specifically, in strongly precessing cases, the directions of $\boldsymbol{L}(t)$ and $\boldsymbol{J}(t)$ can be very different close to merger. As a consequence, the inspiralplunge modes in the inertial $\boldsymbol{J}\left(t_{\Omega \text { peak }}^{\mathrm{EOB}}\right)$ frame can present strong amplitude and frequency oscillations around merger. [Technically those strong oscillations are generated by drastic time-dependent rotations from well-behaved quasinonprecessing inspiral-plunge modes as the merger is approached.] Thus, to keep the matching procedure stable in strongly precessing situations, we set the matching time $t_{\text {match }}^{\ell m}$ earlier and make the matching interval $\Delta t_{\text {match }}^{\ell m}$ longer. We choose

$$
\begin{gathered}
t_{\text {match }}^{\ell m}=t_{\text {match }}^{22, \mathrm{Cal}}-10 M\left(1-\left|\kappa_{L J}\left(t_{\Omega \text { peak }}^{\mathrm{EOB}}\right)\right|\right), \\
\Delta t_{\text {match }}^{\ell m}=\Delta t_{\text {match }}^{22, \mathrm{Cal}}\left(10-9\left|\kappa_{L J}\left(t_{\Omega \text { peak }}^{\mathrm{EOB}}\right)\right|\right),
\end{gathered}
$$

where

$$
\begin{aligned}
t_{\text {match }}^{22, \mathrm{Cal}} & =t_{\Omega \text { peak }}^{E O B}-\left\{\begin{array}{ll}
2.5 M & \chi \leq 0 \\
2.5 M+1.77 M\left(\frac{\chi}{0.437}\right)^{4} & \chi>0
\end{array},\right. \\
\Delta t_{\text {match }}^{22, \mathrm{Cal}} & =7.5 M
\end{aligned}
$$

are the calibrated values of the $(2,2)$ mode in Ref. [13],

$$
\chi=\chi_{S}+\chi_{A} \frac{\sqrt{1-4 \nu}}{1-2 \nu}
$$

is a linear combination of initial spin projections on $\boldsymbol{L}_{N}$, and

$$
\kappa_{L J}\left(t_{\Omega \text { peak }}^{\mathrm{EOB}}\right)=\hat{\boldsymbol{L}}\left(t_{\Omega \text { peak }}^{\mathrm{EOB}}\right) \cdot \hat{\boldsymbol{J}}\left(t_{\Omega \text { peak }}^{\mathrm{EOB}}\right)
$$

is the cosine of the opening angle between the orbital and total angular momenta at the reference time of merger $t_{\Omega \text { peak }}^{\mathrm{EOB}}$. When $\kappa_{L J}\left(t_{\Omega \text { peak }}^{\mathrm{EOB}}\right)=0$, the matching time $t_{\text {match }}^{\ell m}$ is $10 M$ earlier than that of the aligned case, and the time interval $\Delta t_{\text {match }}^{\ell m}$ is 10 times that of the aligned case. The choice of $10 M$ and the factor of 10 made in this paper are rather arbitrary. They are based on the only requirement of producing qualitatively sound merger-ringdown waveforms. We verify in Sec. IV and especially in the comparison with NR waveforms in Sec. IV B that these choices indeed achieve our goal. Further improvements of the matching procedure, which likely go beyond a simple tuning of these parameters ${ }^{6}$, will be carried out when more accurate and strongly precessing NR waveforms become available.

\footnotetext{
${ }^{6}$ The post-merger precession of QNMs observed through NR waveforms in Ref. [67] should be considered, for instance.
} 
TABLE I. We list the binary parameters and number of GW cycles $N_{c y c}$ of the four precessing EOB waveforms that we consider in Sec. IV. Case 3 corresponds to the NR simulation SXS:BBH:0052 of Ref. [46], and case 4 corresponds to SXS:BBH:0058.

\begin{tabular}{lllllccccc}
\hline \hline Case & $q$ & $\chi_{1}$ & $\chi_{2}$ & $\theta_{1}^{S}$ & $\theta_{2}^{S}$ & $\phi_{1}^{S}$ & $\phi_{2}^{S}$ & $M \Omega_{0}$ & $N_{c y c}$ \\
\hline 1 & 2 & 0.6 & 0.6 & $\pi / 3$ & $\pi / 3$ & 0 & $\pi / 2$ & 0.0112 \\
2 & 6 & $0.8^{\mathrm{a}}$ & 0.6 & $\pi / 2$ & $2 \pi / 3$ & $\pi / 2$ & $\pi / 2$ & 0.0112 \\
3 & 3 & 0.5 & 0.5 & $0.5 \pi$ & $0.99 \pi$ & $0.77 \pi$ & $0.31 \pi$ & 0.0177 & 35 \\
4 & 5 & 0.5 & 0 & $0.5 \pi$ & 0 & $-0.79 \pi$ & 0 & 0.0158 & 65 \\
\hline \hline
\end{tabular}

${ }^{\mathrm{a}}$ The nonprecessing EOB model of Ref. [13] generates waveforms with any mass ratio and individual spin magnitudes $-1 \leq \chi_{i} \lesssim 0.7$. Although we consider here $\chi_{1}=0.8$ because $\chi_{1}(t)=\chi_{1} \hat{\boldsymbol{S}}_{1}(t) \cdot \hat{\boldsymbol{L}}_{N}(t)<0.7$ during the entire evolution, we do not find any problem in this case when generating the quasi-nonprecessing waveforms.

\section{COMPARISON BETWEEN PRECESSING WAVEFORMS}

We generate four examples of EOB precessing waveforms using the model defined in Sec. III. The first two examples are a $q=2 \mathrm{BH}$ binary system exhibiting moderate precession-induced modulations and a $q=6$ binary system exhibiting strong modulations. The other two examples are chosen among the 171 NR simulations reported in Ref. [46] with the criterion of long and accurate waveforms exhibiting strong modulations. In these cases we compare NR, PN, and EOB precessing waveforms. The physical parameters of the four binary configurations are listed in Table I.

\section{A. Precessing and radiation-axis frames}

In Sec. III D, we have proposed $\hat{\boldsymbol{L}}_{N}(t)$ and $\hat{\boldsymbol{L}}(t)$ as possible basis vectors for the precessing source frame. In this section, we compare their trajectories and the corresponding precessing waveforms generated through their respective precessing source frames. For convenience, we refer to waveforms generated in these precessing source frames as the $\hat{\boldsymbol{L}}_{N}(t)$-frame and $\hat{\boldsymbol{L}}(t)$-frame waveforms, respectively. Furthermore, we extract the quadrupole-preferred radiation axis [29] from the precessing waveforms and compare their trajectories with either $\hat{\boldsymbol{L}}_{N}(t)$ or $\hat{\boldsymbol{L}}(t)$.

Figure 3 shows for cases 1 and 2 of Table I the trajectories of the unit vectors $\hat{\boldsymbol{J}}, \hat{\boldsymbol{L}}, \hat{\boldsymbol{L}}_{N}, \hat{\boldsymbol{S}}_{1}$, and $\hat{\boldsymbol{S}}_{2}$ in the plane perpendicular to $\boldsymbol{J}\left(t_{\Omega \text { peak }}^{\mathrm{EOB}}\right)$. In both cases, the BHs complete more than two cycles of precession, and the directions of $\boldsymbol{J}(t)$ are well conserved during the entire inspiral phase. All other vectors precess around $\boldsymbol{J}(t)$. These are expected features of the well-known simple-precession picture of spinning binaries in PN theory [23]. Another common feature in both cases is the difference between the trajectories of $\boldsymbol{L}_{N}(t)$ and $\boldsymbol{L}(t)$. The trajectory of $\boldsymbol{L}_{N}(t)$ shows nutation at twice the orbital frequency, and its average follows the smooth precession trajectory of $\boldsymbol{L}(t)$. From PN theory [49]

$$
\boldsymbol{L}=\boldsymbol{L}_{N}+\boldsymbol{L}_{P N}+\boldsymbol{L}_{S O}+\mathcal{O}\left(c^{-4}\right),
$$

where

$$
\boldsymbol{L}_{P N} \equiv \boldsymbol{L}_{N}\left[\frac{1}{2} v^{2}(1-3 \nu)+(3+\nu) \frac{M}{r}\right]
$$

$$
\boldsymbol{L}_{S O} \equiv-\frac{2 \mu}{r}\left[\left(\boldsymbol{S}_{e f f} \cdot \hat{\boldsymbol{L}}_{N}\right) \hat{\boldsymbol{L}}_{N}+\left(\boldsymbol{S}_{e f f} \cdot \hat{\lambda}\right) \hat{\lambda}\right],
$$

with $v \equiv \hat{\Omega}^{1 / 3}, \hat{\lambda} \equiv\left(\hat{\boldsymbol{L}}_{N} \times \boldsymbol{r}\right) / r$ and

$$
\boldsymbol{S}_{\mathrm{eff}} \equiv\left(1+\frac{3 m_{2}}{4 m_{1}}\right) \boldsymbol{S}_{1}+\left(1+\frac{3 m_{1}}{4 m_{2}}\right) \boldsymbol{S}_{2} .
$$

Note that the unit vector $\hat{\boldsymbol{\lambda}}$ instantaneously rotates about $\hat{\boldsymbol{L}}_{N}$ at the orbital frequency $\Omega$. In addition, $\boldsymbol{L}$ obeys a simple precession equation about $\boldsymbol{J}$, i.e., $\boldsymbol{L} \propto \boldsymbol{J} \times \boldsymbol{L}$ (see Eq. (2.13) of Ref. [49]). This, together with Eq. (28), implies that $\boldsymbol{L}_{N}$ cannot simply precess about $\boldsymbol{J}$. When computing $\hat{\boldsymbol{L}}_{N}$, the spin-orbit term $\boldsymbol{L}_{S O}$ generates contributions of the form

$$
\left(\boldsymbol{S}_{\mathrm{eff}} \cdot \dot{\hat{\lambda}}\right) \hat{\lambda} \quad \text { and } \quad\left(\boldsymbol{S}_{\mathrm{eff}} \cdot \hat{\lambda}\right) \dot{\hat{\lambda}}
$$

which indeed oscillate at twice the orbital frequency, accounting for the nutations seen in Fig. 3. Note that this behavior is consistent with the observation of Ref. [29] while it does not agree with Ref. [68], where the authors used orbit-averaged formula to describe the precession of $\boldsymbol{L}_{N}(t)$.

The main difference between the two cases is the size of the opening angle between $\boldsymbol{J}(t)$ and $\boldsymbol{L}(t)$ and correspondingly the strength of the orbital precession. In the comparable-mass $q=2$ case, $\boldsymbol{L}(t)$ always dominates over the $\mathrm{BH}$ spins during inspiral, and the angle between $\boldsymbol{J}(t)$ and $\boldsymbol{L}(t)$ remains small. The orbital precession is therefore mild. In the $q=6$ case, on the contrary, the contribution of $\boldsymbol{S}_{1}(t)$ to $\boldsymbol{J}(t)$ is comparable to that of $\boldsymbol{L}(t)$ initially and becomes more and more dominant. Because of the large opening angle between $\boldsymbol{J}(t)$ and $\boldsymbol{L}(t)$, the direction of $\boldsymbol{L}(t)$ changes more than $\pi / 2$ during precession, and an initially face-on binary becomes edge-on a few times during the inspiral.

In Fig. 4, we compare precessing waveforms generated in the $\hat{\boldsymbol{L}}_{N}(t)$ and $\hat{\boldsymbol{L}}(t)$ precessing source frames. Considering the oscillatory difference between 

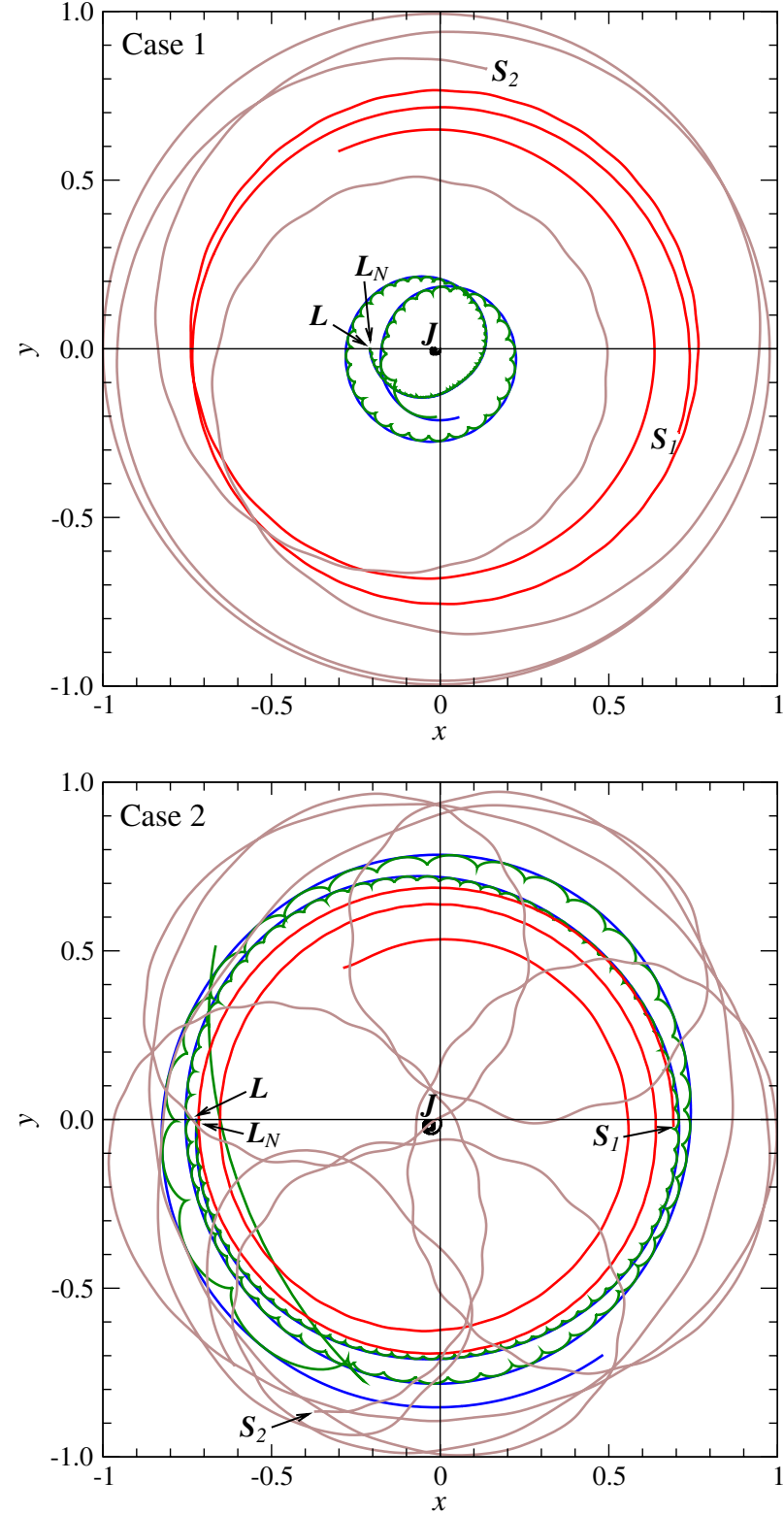

FIG. 3 (color online). We show the projections of $\hat{\boldsymbol{J}}(t), \hat{\boldsymbol{L}}(t)$, $\hat{\boldsymbol{L}}_{N}(t), \hat{\boldsymbol{S}}_{1}(t)$, and $\hat{\boldsymbol{S}}_{2}(t)$ on the $x-y$ plane of the inertial frame for which the $z$ axis is aligned with $\boldsymbol{J}\left(t_{\Omega \text { peak }}^{\mathrm{EOB}}\right)$. In the top and bottom panels, we show trajectories of these unit vectors for cases 1 and 2 of Table I, respectively. The initial point of each trajectory is marked by its name. The trajectory of $\hat{\boldsymbol{J}}(t)$ ends at the origin, by definition. The trajectory of $\hat{\boldsymbol{L}}_{N}(t)$ follows that of $\hat{\boldsymbol{L}}(t)$ with oscillations due to nutation.

the trajectories of $\hat{\boldsymbol{L}}_{N}(t)$ and $\hat{\boldsymbol{L}}(t)$ shown in Fig. 3, it is somewhat unexpected that the precessing waveforms agree quite well. In case 1 , the waveforms are visually indistinguishable during inspiral-with the relative amplitude difference below $1 \%$ and phase difference below $0.02 \mathrm{rad}$. Even in the $q=6$ case 2, where precession is strong, the waveforms agree reasonably well. Although the relative amplitude and phase differences oscillate strongly when the amplitudes of the waveforms are small, their averages differ only by $<5 \%$ and $<0.15$ rad over the $\sim 24000 M$ long inspiral. The oscillations are due to the precession-induced modulation and are expected to be strong when the orbital plane goes through a nearly edge-on phase, corresponding to small waveform amplitudes.

In Fig. 5, we examine closely the waveforms as well as their phase evolutions over a time period of $500 \mathrm{M}$. The real and imaginary parts of $h_{22}$, i.e., its + and $\times$ polarizations in the radiation frame, show substantial amplitude difference, implying a deviation from circular polarization due to the orbital plane inclination. The phase evolves most rapidly when the dominant quadrature (the imaginary part in this example) goes through zero. Even a small difference in the times when this happens for the two waveforms leads to a burst of phase difference. Such phase differences can be partly removed by time shifting the two waveforms, but not through a phase shift. In spite of these bursts of amplitude and phase difference, the overall agreement of the waveforms is good. The overlaps between the $\hat{\boldsymbol{L}}_{N}(t)$-frame and $\hat{\boldsymbol{L}}(t)$-frame waveforms, optimized over time and phase of coalescence, are above 0.999 in case 1 and above 0.985 in case $2^{7}$. The lower overlaps in case 2 are due to the larger difference between $\hat{\boldsymbol{L}}_{N}(t)$-frame and $\hat{\boldsymbol{L}}(t)$-frame waveforms during merger and ringdown.

Finally, we examine the preferred radiation axis determined by the waveforms extracted at infinity. Since we developed only the $\ell=2$ modes in the current EOB model, we calculate the quadrupole-preferred radiation axis [29] with a small modification. In Ref. [29], the quadrupolepreferred axis is determined by maximizing the power in the $(2,2)$ and $(2,-2)$ modes of the Weyl scalar $\Psi_{4}(t)$. We determine the quadrupole-preferred axis by maximizing the power in the strain modes $h_{22}(t)$ and $h_{2,-2}(t)$. Specifically, given the $h_{2 m}^{(o)}(t)$ modes in an arbitrary original frame, the $h_{2 m}^{(n)}(t)$ modes in a new frame are given by Eq. (7); so we compute the Euler angles $\alpha(t), \beta(t)$, and $\gamma(t)$ (defining the rotation from the original to the new frame) that maximize the quantity $\left|h_{22}^{(n)}(t)\right|^{2}+\left|h_{2,-2}^{(n)}(t)\right|^{2}$. The quadrupolepreferred axis is then given by the $z$ axis of the new frame defined by these Euler angles. We find that the quadrupolepreferred axis computed from $\hat{\boldsymbol{L}}_{N}(t)$-frame or $\hat{\boldsymbol{L}}(t)$-frame waveforms agrees with $\hat{\boldsymbol{L}}_{N}(t)$ or $\hat{\boldsymbol{L}}(t)$ to within $0.3^{\circ}$ during inspiral. That is to say, the preferred radiation axis determined by EOB waveforms coincides with the reference axis $\left[\hat{\boldsymbol{L}}_{N}(t)\right.$ or $\left.\hat{\boldsymbol{L}}(t)\right]$ of the precessing frame determined by the EOB dynamics. Therefore, comparisons of preferred radiation axes determined by $\mathrm{NR}$ and $\mathrm{EOB}$ precessing waveforms will provide direct information for calibrating the precession dynamics, in particular the dynamics of $\hat{\boldsymbol{L}}_{N}(t)$ and $\hat{\boldsymbol{L}}(t)$, of the EOB model.

\footnotetext{
${ }^{7}$ The overlaps are calculated using the zero-detuned highpower Advanced LIGO noise curve [3] for the range of binary total masses from 20 to $200 M_{\odot}$.
} 

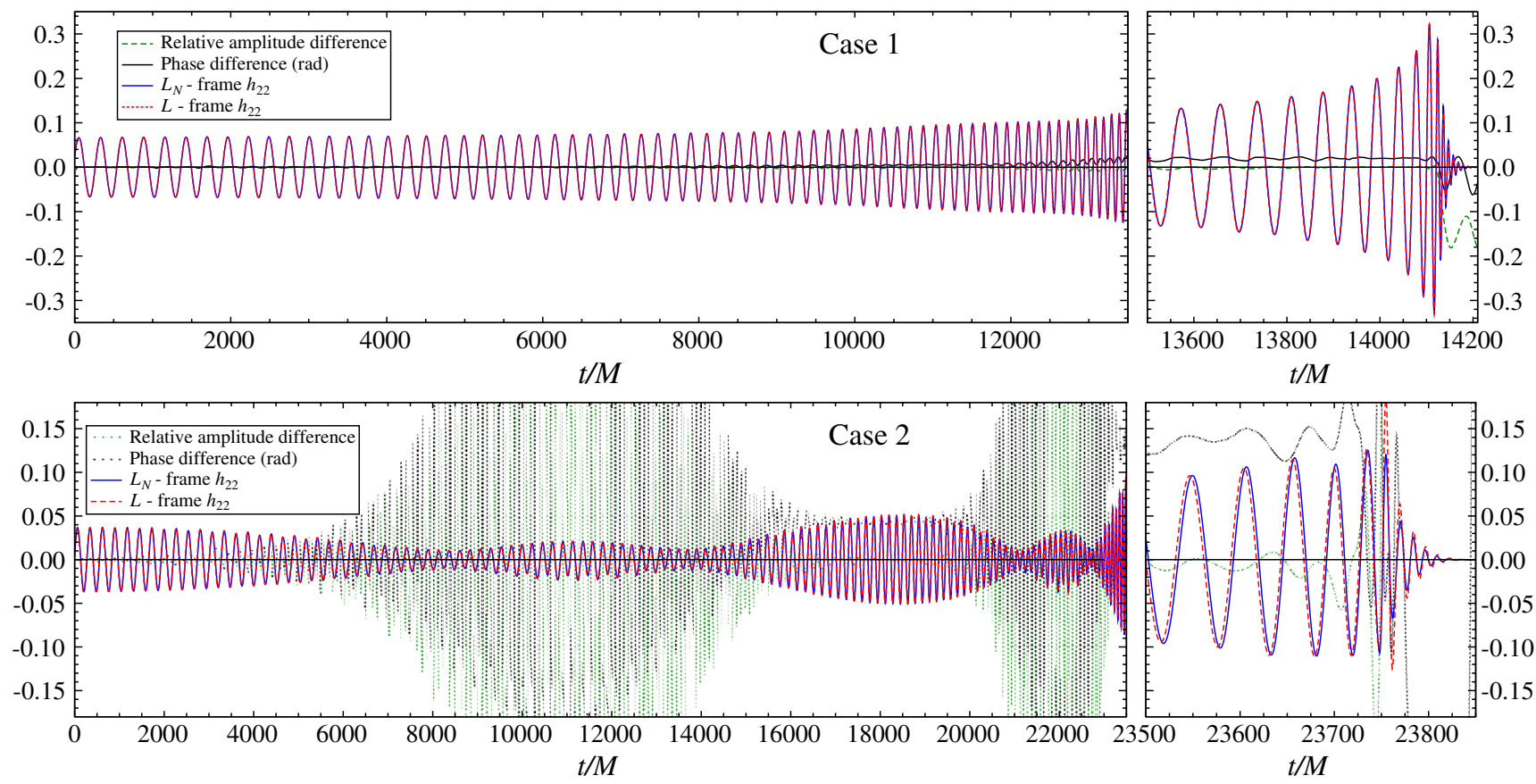

FIG. 4 (color online). We show the $\hat{\boldsymbol{L}}_{N}(t)$-frame and the $\hat{\boldsymbol{L}}(t)$-frame precessing waveforms, as well as their relative amplitude and phase differences. The top and bottom panels are waveforms for cases 1 and 2 of Table I. The left and right panels show the inspiral and the plunge-merger-ringdown stages of the waveforms, respectively.

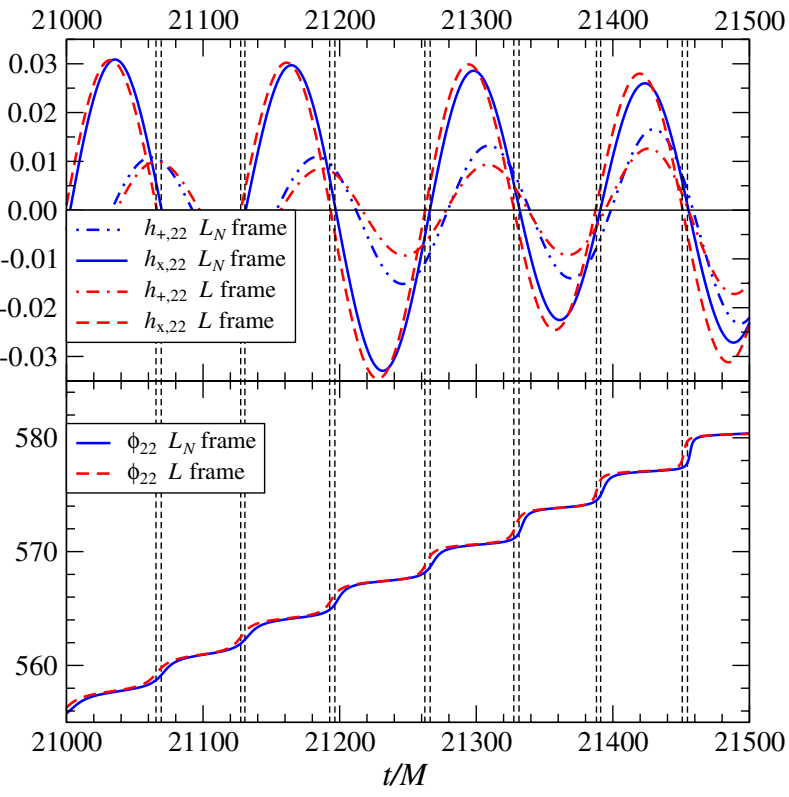

FIG. 5 (color online). For case 2 of Table I, we show the $\hat{\boldsymbol{L}}_{N}(t)$ frame and $\hat{\boldsymbol{L}}(t)$-frame waveforms in the top panel and their phase evolutions in the bottom panel, over a short time period from $t=21000 M$ to $21500 M$. The vertical lines mark the time when the dominant quadrature (the imaginary part for this specific instance) of any waveform becomes zero. It coincides with the time when the corresponding phase evolution in the bottom panel experiences a rapid growth. The absolute phase values are not relevant.

\section{B. Comparison with numerical-relativity waveforms}

The precessing EOB model defined in Sec. III is not calibrated to any precessing numerical simulations. The only nonperturbative information extracted from NR simulations and employed in this precessing EOB model is contained in the spinning, nonprecessing sector, which was calibrated to only two equal-mass, spinning, nonprecessing numerical simulations [44] and five nonspinning ones [42,45] in Ref. [13]. It is therefore highly interesting to compare the EOB precessing waveforms to NR waveforms.

The Caltech-Cornell-CITA collaboration has recently produced a large number of long and accurate waveforms [46]. We choose among them two precessing waveforms that are sufficiently long ( $\sim 35$ and $\sim 65$ GW cycles) and display strong precessional modulations. The physical parameters of these two waveforms are given in the last two rows of Table I. We compare those numerical waveforms also with the PN SpinTaylorT4 (ST4) inspiraling waveforms [25], which are commonly used in the literature and in LIGO and Virgo software. We generate the ST4 waveforms at the highest PN order available today, namely, spin-amplitude corrections through $1.5 \mathrm{PN}$ order $[47]^{8}$ and

\footnotetext{
${ }^{8}$ The 2PN spin-amplitude corrections were derived in Ref. [22]. Since they are not yet implemented in any ready-to-use software package and are not crucial for the purpose of our comparisons, we do not include them here.
} 
phase corrections through 3.5PN order [21] using the LIGO Algorithm Library [69].

We extract the initial values of $\boldsymbol{S}_{1}, \boldsymbol{S}_{2}$, and GW frequency from the NR data soon after the junk radiation, which typically carries away unphysical radiation present in the initial data. We then set EOB and ST4 initial conditions using these values and start their evolutions after the junk-radiation time, which is $t=230 \mathrm{M}$ for case 3 of Table I and $t=160 M$ for case 4 of Table I. We align the orbital orientation $\hat{\boldsymbol{L}}_{N}$ at these after-junkradiation times with the inertial source frame $\left\{\boldsymbol{e}_{1}^{S}, \boldsymbol{e}_{2}^{S}, \boldsymbol{e}_{3}^{S}\right\}$ (see Fig. 1) and use it as the default frame for our comparisons. Unlike the case of nonprecessing dynamics and waveforms, we must impose specific $\boldsymbol{S}_{1}$ and $\boldsymbol{S}_{2}$ directions relative to the initial binary separation vector $\boldsymbol{r}_{0}$ at a specific after-junk-radiation time. Thus, we do not apply any time or phase shifts when comparing numerical and analytical waveforms.

In Fig. 6 we compare the evolutions of the dimensionless spin vectors $\chi_{1}(t)=\boldsymbol{S}_{1}(t) / m_{1}^{2}$ and $\chi_{2}(t)=\boldsymbol{S}_{2}(t) / m_{2}^{2}$ $\left(\chi_{2}=0\right.$ for case 4$)$ for the NR, EOB, and ST4 dynamics. Quite remarkably, the EOB spins follow the NR ones rather accurately all the way through the inspiral-plunge stage, while the ST4 spins, although capturing the qualitative precessional behavior of the NR ones, show quantitative differences in both the inspiral and precessional time scales.

In Figs. 7 and 8, we compare NR, EOB, and ST4 $h_{22}$ modes decomposed in the inertial source frame $\left\{\boldsymbol{e}_{1}^{S}, \boldsymbol{e}_{2}^{S}, \boldsymbol{e}_{3}^{S}\right\}$. Since the source frame is aligned with the initial orbital orientation $\left[\hat{\boldsymbol{L}}_{N}\right]_{0}$ and the binary orbit precesses only moderately in case 3 , there are only moderate modulations on $h_{22}$ in this case. The modulations in case 4 are strong, though. In both cases, the agreement between NR and EOB $h_{22}$ modes is remarkable. Their amplitudes agree quite well, and their phases, aligned at the initial time, differ by only $\sim 0.2 \mathrm{rad}$ at merger, i.e., at the peak of the NR $(2,2)$ mode. The agreement between NR and ST4 $h_{22}$ modes, although not comparable with the agreement between NR and EOB, is also very good. Even though the amplitudes differ by $\sim 10 \%$ during the inspiral, because amplitude corrections are known only through $1.5 \mathrm{PN}$ order in the spinning case [47], their phases agree quite well for tens of cycles but start departing from each other $10 \mathrm{GW}$ cycles before merger. Quite interestingly, we have found that using the newly available 3.5PN spin-orbit effects [21] in the phasing of ST4 improves the agreement with the NR waveforms. If we were using the $2.5 \mathrm{PN}$ phasing, the end of the inspiral would occur at $\sim 460 M(960 M)$ instead of $\sim 60 M(140 M)$ after the merger of the NR waveform, for case 3 (4). Moreover, we find for cases 3 and 4 that when we align the 3.5PN and NR phasing at the after-junkradiation time they accumulate a difference of $1 \mathrm{GW}$ cycle only 1 cycle before merger. By contrast the $2.5 \mathrm{PN}$
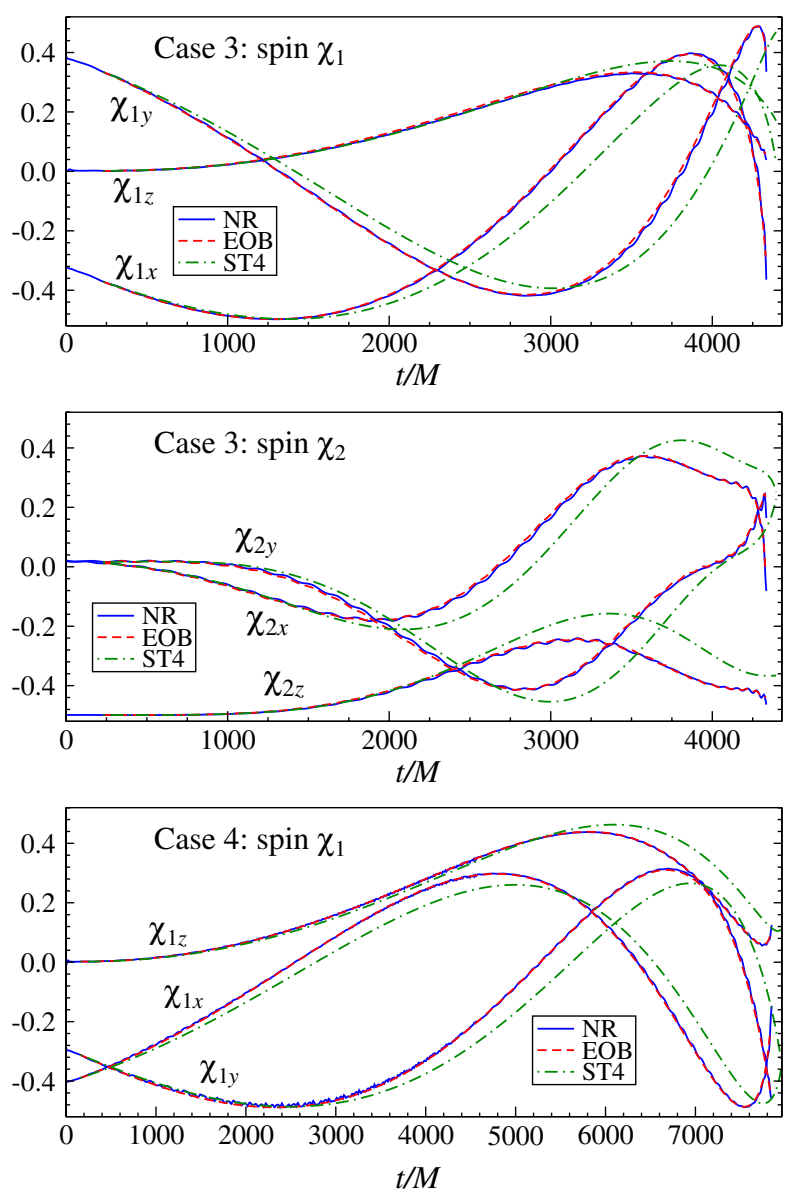

FIG. 6 (color online). We show evolutions of the dimensionless spin vectors $\chi_{1}=S_{1}(t) / m_{1}^{2}$ and $\chi_{2}=S_{2}(t) / m_{2}^{2}$ of the NR simulation and the EOB and ST4 models. Specifically, we show the projections of $\chi_{1}$ and $\chi_{2}$ on the basis vectors of the inertial source frame $\left\{\boldsymbol{e}_{1}^{S}, \boldsymbol{e}_{2}^{S}, \boldsymbol{e}_{3}^{S}\right\}$ that is aligned with the initial orbital orientation $\left[\hat{\boldsymbol{L}}_{N}\right]_{0}$ (see . 1). The top two panels show $\chi_{1}$ and $\chi_{2}$ for case 3 of Table I. The bottom panel shows $\chi_{1}\left(\chi_{2}=0\right)$ for case 4 of Table I. The EOB and ST4 data start at the after-junk-radiation time in the NR simulations, which are $t=230 M$ and $t=160 M$ for cases 3 and 4, respectively.

phasing differs from the NR phasing by $1 \mathrm{GW}$ after 28 (37) GW cycles [or 6 (16) GW cycles before merger] for case 3 (4).

The agreement between NR and EOB modes $(2,1)$ and $(2,0)$ modes is also very good. Rather than the modes, we show in Fig. 9 the NR and EOB polarizations $h_{+}(t)$ given by Eq. (3). Since only the $\ell=2$ modes are available in the current precessing EOB model, we limit the summation over $\ell$ to only $\ell=2$. To include substantial contributions from all $\ell=2$ modes, we choose $\theta=\pi / 3$ and $\phi=\pi / 2$ for the direction of GW propagation $N$ (see Fig. 1). As expected from the very good agreement of the individual modes, the NR and EOB polarizations also agree remarkably.

Finally, we measure the difference between EOB and NR polarizations with the unfaithfulness [70], defined as 


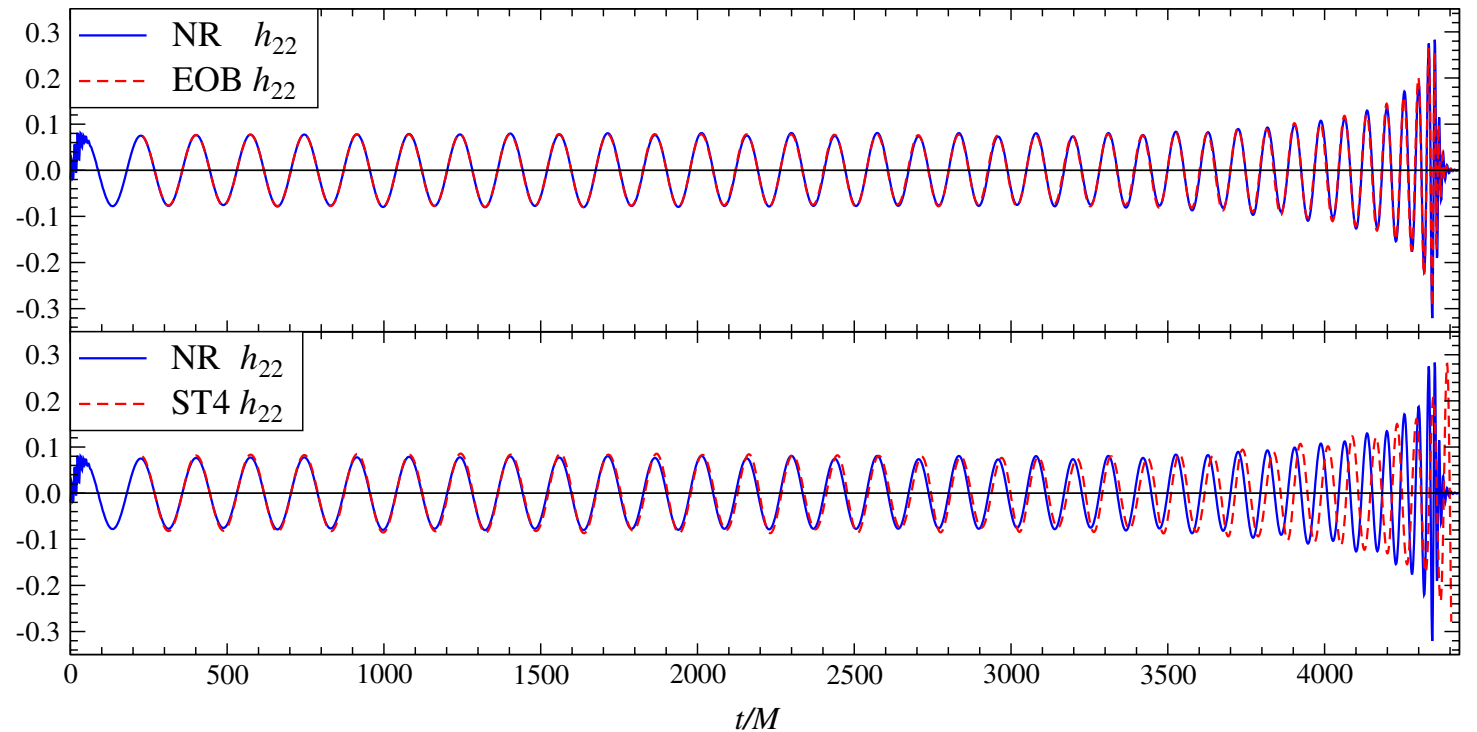

FIG. 7 (color online). We show for case 3 of Table I the $h_{22}$ mode decomposed in the inertial source frame $\left\{\boldsymbol{e}_{1}^{S}, \boldsymbol{e}_{2}^{S}, \boldsymbol{e}_{3}^{S}\right\}$ that is aligned with the initial orbital orientation $\left[\hat{\boldsymbol{L}}_{N}\right]_{0}$ (see . 1). For clarity, we show the NR and EOB $h_{22}$ in the top panel and the NR and ST4 $h_{22}$ in the bottom panel. The EOB and ST4 data start at the after-junk-radiation time of $t=230 \mathrm{M}$.

$$
\overline{\mathcal{F}}=1-\max _{t_{c}, \phi_{c}, \psi} \frac{\left\langle h_{\mathrm{NR}} \mid h_{\mathrm{EOB}}\right\rangle}{\sqrt{\left\langle h_{\mathrm{NR}} \mid h_{\mathrm{NR}}\right\rangle\left\langle h_{\mathrm{EOB}} \mid h_{\mathrm{EOB}}\right\rangle}},
$$

where the EOB waveform of the detector response is

$$
\begin{aligned}
h_{\mathrm{EOB}}\left(t ; t_{c}, \phi_{c}, \psi, \lambda\right) \propto & \cos \psi h_{\mathrm{EOB},+}\left(t ; t_{c}, \phi_{c}, \boldsymbol{\lambda}\right) \\
& +\sin \psi h_{\mathrm{EOB}, \times}\left(t ; t_{c}, \phi_{c}, \boldsymbol{\lambda}\right)
\end{aligned}
$$

and the maximization is over the time and phase of coalescence $t_{c}$ and $\phi_{c}$, as well as the polarization angle $\psi$ that combines the + and $\times$ polarizations in the radiation frame. We do not optimize over the physical binary parameters $\lambda$; i.e., we use the same $\lambda$ in $h_{\mathrm{NR}}$ and $h_{\mathrm{EOB}}$. Note that since we include modes with different $m, \phi_{c}$ and $\psi$ are no longer degenerate, and both of them have to be maximized over. We define the inner product between two waveforms through the integral in the frequency domain

$$
\left\langle h_{1}, h_{2}\right\rangle \equiv 4 \operatorname{Re} \int_{0}^{\infty} \frac{\tilde{h}_{1}(f) \tilde{h}_{2}^{*}(f)}{S_{h}(f)} d f
$$

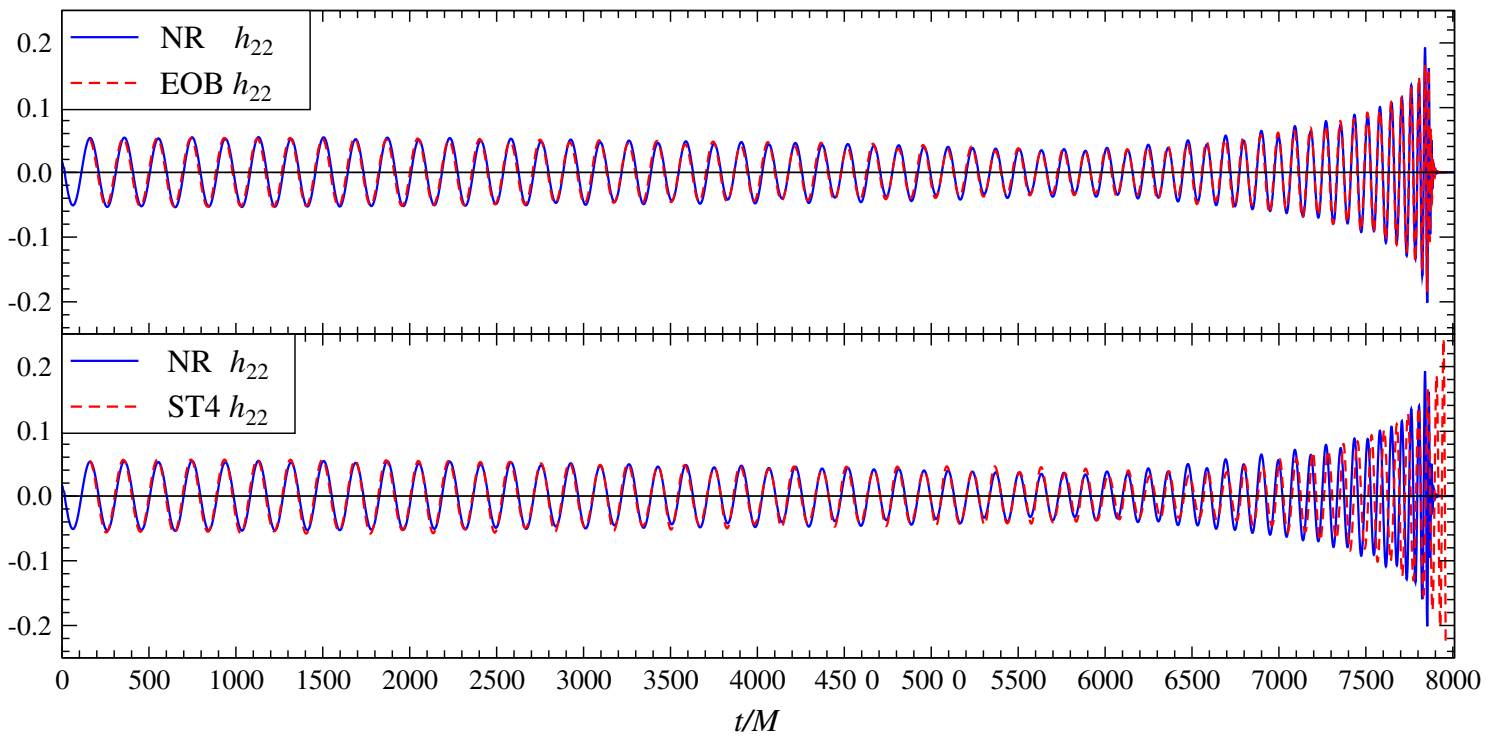

FIG. 8 (color online). We show for case 4 of Table I the $h_{22}$ mode decomposed in the inertial source frame $\left\{\boldsymbol{e}_{1}^{S}, \boldsymbol{e}_{2}^{S}, \boldsymbol{e}_{3}^{S}\right\}$ that is aligned with the initial orbital orientation $\left[\hat{\boldsymbol{L}}_{N}\right]_{0}$ (see . 1). For clarity, we show the NR and EOB $h_{22}$ in the top panel and the NR and ST4 $h_{22}$ in the bottom panel. The EOB and ST4 data start at the after-junk-radiation time of $t=160 \mathrm{M}$. 

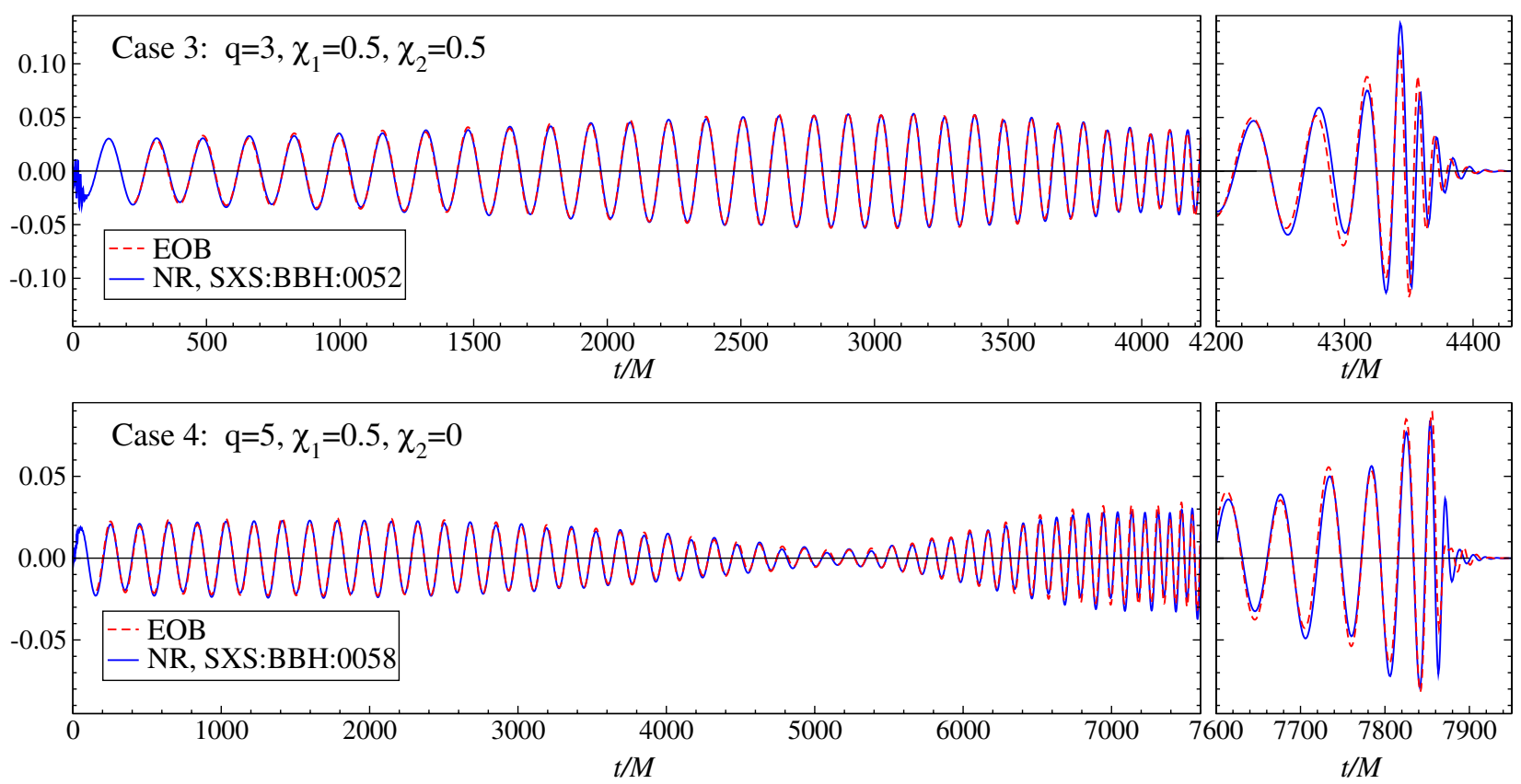

FIG. 9 (color online). We show for cases 3 and 4 of Table I the GW polarization $h_{+}$, containing contributions from $\ell=2$ modes, that propagates along a direction $\hat{N}$ specified by spherical coordinates $\theta=\pi / 3$ and $\phi=\pi / 2$ associated with the inertial source frame $\left\{\boldsymbol{e}_{1}^{S}, \boldsymbol{e}_{2}^{S}, \boldsymbol{e}_{3}^{S}\right\}$. The EOB waveforms start at the after-junk-radiation times of $t=230 M$ and $t=160 M$, respectively.

where $\tilde{h}_{1}(f)$ and $\tilde{h}_{2}(f)$ are frequency-domain waveforms and $S_{h}(f)$ is the noise power spectral density of the detector. We employ the zero-detuned high-power advanced LIGO noise curve ZERO_DET_HIGH_P given in Ref. [3]. The NR waveforms, although very long, cover the entire advanced LIGO frequency band only for $M \geq 100 M_{\odot}$. Thus, to reduce artifacts when considering binaries with $M<100 M_{\odot}$, we taper both ends of the NR and EOB waveforms using the Planck-taper window function [71] (see Ref. [12] for details). In Fig. 10, we show the EOB unfaithfulness when the total mass $M$ varies between $20 M_{\odot}$ and $200 M_{\odot}$. We choose the same direction of GW propagation $N$ as is considered in Fig. 9, namely, $\theta=\pi / 3$ and $\phi=\pi / 2$.

For each waveform we estimate the numerical error in the unfaithfulness results of Fig. 10 by calculating the unfaithfulness of the EOB waveform with two numerical waveforms: the extrapolated high-resolution waveform shown in Fig. 9 and the outermost finite-radius highresolution waveform. We use the difference between these unfaithfulness results to estimate the extrapolation error. We might estimate the finite resolution errors in the same way by calculating the unfaithfulness of the EOB waveform with the extrapolated high- and medium-resolution numerical waveforms. However, medium-resolution simulations for these two cases are not available, but we expect from previous studies that errors due to resolution are smaller than errors due to extrapolation [12].

Since the unfaithfulness of EOB waveforms is below $\sim 2 \%$, we expect that the ineffectualness, which measures the difference between EOB and NR waveforms when minimizing also over the binary parameters $\lambda$, will be below $1 \%$ (with a loss of event rates less than $3 \%$ ). Thus, for those two precessing binary configurations, the EOB waveforms are sufficiently accurate for detection with advanced LIGO detectors.

Although these very encouraging results refer only to two precessing binary configurations, they strongly suggest

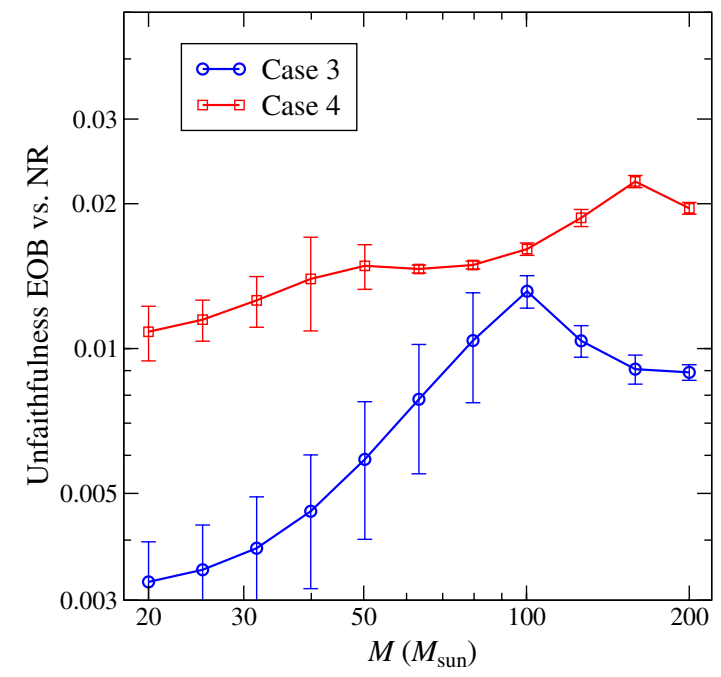

FIG. 10 (color online). Unfaithfulness of the EOB waveforms when compared to NR waveforms as a function of the total binary mass. Shown are cases 3 and 4 of Table I. The error bars are estimates of numerical errors. The direction of GW propagation $\hat{N}$ is specified by the spherical coordinates $\theta=\pi / 3$ and $\phi=\pi / 2$. 
that the approach we have proposed for modeling precessing compact binaries within the EOB model is bound to succeed. A more comprehensive and careful comparison of the EOB model with a larger number of accurate NR simulations will be carried out in the near future using the entire catalog of simulations in Ref. [46].

\section{CONCLUSIONS}

So far, the EOB modeling of GWs emitted from compact binaries has focused primarily on nonprecessing binary configurations [6-14]. Nonspinning EOB waveforms have been employed in the first searches of GWs from high-mass binary BHs with LIGO and Virgo detectors [72-74]. Recently, studies carried out within the NRAR collaboration [19] have shown that nonprecessing EOB waveforms originally calibrated to seven NR waveforms $[42,44,45]$ in Ref. [13] match very well also tens of new NR waveforms produced within the NRAR collaboration. The next, challenging, task is to achieve a similar success also for generic, spinning binary configurations. In this paper we have started addressing this important problem.

Building on previous work [11,13,25,38-41], we have proposed a strategy to generate EOB precessing waveforms. The procedure employs the precessing convention of Ref. [25] that minimizes the precession-induced modulations in the waveform's phase and amplitude and an inertial frame aligned with the spin of the final $\mathrm{BH}$ where the matching between the inspiral-plunge and mergerringdown EOB waveforms is carried out.

When spins are aligned or antialigned with the orbital angular momentum, the EOB precessing waveforms that we have built reduce to the nonprecessing $\mathrm{EOB}$ waveforms calibrated to seven nonprecessing NR waveforms in Ref. [13]. Since the factorized energy flux is not yet available for precessing spins, we have included in the radiation-reaction force of the EOB dynamics only spin couplings for which the projection along the orbital angular momentum is different from zero. This limitation will be relaxed in the future as soon as the radiation-reaction sector of the EOB model is improved. Furthermore, we have limited this first study to the EOB $\ell=2$ modes.

Without recalibrating the EOB precessing waveforms, we have then compared them to two, long, strongly precessing NR waveforms that were recently produced in Ref. [46]. We have found a remarkable agreement both for the dynamics, that is, the spins' components, and the gravitational polarizations. In particular, when using the advanced-LIGO noise spectral density, the mismatches between the EOB and NR waveforms for binary masses
$20-200 M_{\odot}$ are below $2 \%$ when maximizing only on the time and phase at coalescence and on the polarization angle. Although those results only refer to two binary configurations, they are very encouraging and suggest that the EOB precessing model developed here is an excellent starting point for building a generic, spinning EOB model for advanced LIGO and Virgo searches. We have also compared the two NR waveforms to PN ST4 waveforms that are largely used in the literature and in LIGO and Virgo software. We have found that the PN waveforms at 3.5PN order agree very well with NR waveforms for several GW cycles and accumulate a phase difference of $\sim 6 \mathrm{rad}$, starting about $10 \mathrm{GW}$ cycles before merger.

Finally, several analyses were left out in this first study of precessing waveforms. They include (i) a more detailed comparison between spin variables in the numerical simulations and analytical models, (ii) the extension of precessing waveforms to modes higher than $\ell=2$, (iii) a more systematic way of identifying the initial conditions in the numerical and analytical waveforms, and (iv) the inclusion of resolution errors when estimating numerical errors. We defer those important extensions to a future publication where many more NR waveforms will be also analyzed.

\section{ACKNOWLEDGMENTS}

We thank Anıl Zenginoğlu, Geoffrey Lovelace, and Mike Boyle for their contributions to the productions of the NR waveforms used in this paper. A. B., Y. P., and A. T. acknowledge partial support from NSF Grants No. PHY0903631 and No. PHY-1208881. A. B. also acknowledges partial support from the NASA Grant No. NNX12AN10 G. A.M. and H.P. gratefully acknowledge support from NSERC of Canada, the Canada Chairs Program, and the Canadian Institute for Advanced Research. L. K. gratefully acknowledges support from the Sherman Fairchild Foundation and from NSF Grants No. PHY-0969111 and No. PHY-1005426. Simulations used in this work were computed with the SpEC code [75]. Computations were performed on the Zwicky cluster at Caltech, which is supported by the Sherman Fairchild Foundation and by NSF Award No. PHY-0960291, on the NSF XSEDE network under Grant No. TG-PHY990007 N, and on the GPC supercomputer at the SciNet HPC Consortium [76]. SciNet is funded by the Canada Foundation for Innovation under the auspices of Compute Canada, the Government of Ontario, Ontario Research Fund-Research Excellence, and the University of Toronto. 
[1] F. Acernese et al., Classical Quantum Gravity 25, 114045 (2008).

[2] H. Grote (LIGO Scientific Collaboration), Classical Quantum Gravity 25, 114043 (2008).

[3] D. Shoemaker (LIGO Collaboration), LIGO Document T0900288-v3, URL https://dcc.ligo.org/cgi-bin/DocDB/ ShowDocument?docid $=2974$.

[4] J. Aasi et al. (LIGO Scientific Collaboration, Virgo Collaboration), arXiv:1304.0670.

[5] ESA LISA website, http://sci.esa.int/lisa.

[6] A. Buonanno, Y. Pan, J. Baker, J. Centrella, B. Kelly, S. McWilliams, and J. van Meter, Phys. Rev. D 76, 104049 (2007).

[7] T. Damour, A. Nagar, E. N. Dorband, D. Pollney, and L. Rezzolla, Phys. Rev. D 77, 084017 (2008).

[8] T. Damour, A. Nagar, M. Hannam, S. Husa, and B. Bruegmann, Phys. Rev. D 78, 044039 (2008).

[9] A. Buonanno, Y. Pan, H. Pfeiffer, M. Scheel, L. Buchman, and L. Kidder, Phys. Rev. D 79, 124028 (2009).

[10] T. Damour and A. Nagar, Phys. Rev. D 79, 081503 (2009).

[11] Y. Pan, A. Buonanno, L. T. Buchman, T. Chu, L. E. Kidder, H. P. Pfeiffer, and M. A. Scheel, Phys. Rev. D 81, 084041 (2010).

[12] Y. Pan, A. Buonanno, M. Boyle, L. T. Buchman, L. E. Kidder, H. P. Pfeiffer, and M. A. Scheel, Phys. Rev. D 84, 124052 (2011).

[13] A. Taracchini, Y. Pan, A. Buonanno, E. Barausse, M. Boyle, T. Chu, G. Lovelace, H. P. Pfeiffer, and M. A. Scheel, Phys. Rev. D 86, 024011 (2012).

[14] T. Damour, A. Nagar, and S. Bernuzzi, Phys. Rev. D 87, 084035 (2013).

[15] P. Ajith et al., Classical Quantum Gravity 24, S689 (2007).

[16] P. Ajith et al., Phys. Rev. D 77, 104017 (2008).

[17] P. Ajith et al., Phys. Rev. Lett. 106, 241101 (2011).

[18] L. Santamaria et al., Phys. Rev. D 82, 064016 (2010).

[19] I. Hinder et al., (The NRAR Collaboration), Classical Quantum Gravity 31, 025012 (2014).

[20] L. Blanchet, A. Buonanno, and G. Faye, Phys. Rev. D 84, 064041 (2011).

[21] A. Bohe, S. Marsat, and L. Blanchet, Classical Quantum Gravity 30, 135009 (2013).

[22] A. Buonanno, G. Faye, and T. Hinderer, Phys. Rev. D 87, 044009 (2013).

[23] T. A. Apostolatos, C. Cutler, G. J. Sussman, and K. S. Thorne, Phys. Rev. D 49, 6274 (1994).

[24] T. Apostolatos, Phys. Rev. D 52, 605 (1995).

[25] A. Buonanno, Y.-b. Chen, and M. Vallisneri, Phys. Rev. D 67, 104025 (2003).

[26] A. Buonanno, Y. Chen, Y. Pan, H. Tagoshi, and M. Vallisneri, Phys. Rev. D 72, 084027 (2005).

[27] P. Schmidt, M. Hannam, and S. Husa, Phys. Rev. D 86, 104063 (2012).

[28] Y. Pan, A. Buonanno, Y.-b. Chen, and M. Vallisneri, Phys. Rev. D 69, 104017 (2004).

[29] P. Schmidt, M. Hannam, S. Husa, and P. Ajith, Phys. Rev. D 84, 024046 (2011).

[30] R. O'Shaughnessy, B. Vaishnav, J. Healy, Z. Meeks, and D. Shoemaker, Phys. Rev. D 84, 124002 (2011).

[31] M. Boyle, R. Owen, and H. P. Pfeiffer, Phys. Rev. D 84, 124011 (2011).
[32] M. Boyle, Phys. Rev. D 87, 104006 (2013).

[33] L. Pekowsky, R. O'Shaughnessy, J. Healy, and D. Shoemaker, Phys. Rev. D 88, 024040 (2013).

[34] A. Buonanno and T. Damour, Phys. Rev. D 59, 084006 (1999).

[35] A. Buonanno and T. Damour, Phys. Rev. D 62, 064015 (2000).

[36] T. Damour, P. Jaranowski, and G. Schaefer, Phys. Rev. D 62 , 084011 (2000).

[37] T. Damour, Phys. Rev. D 64, 124013 (2001).

[38] A. Buonanno, Y. Chen, and T. Damour, Phys. Rev. D 74, 104005 (2006).

[39] E. Barausse, E. Racine, and A. Buonanno, Phys. Rev. D 80, 104025 (2009).

[40] E. Barausse and A. Buonanno, Phys. Rev. D 81, 084024 (2010).

[41] E. Barausse and A. Buonanno, Phys. Rev. D 84, 104027 (2011).

[42] M. A. Scheel, M. Boyle, T. Chu, L. E. Kidder, K. D. Matthews, and H. P. Pfeiffer, Phys. Rev. D 79, 024003 (2009).

[43] M. Boyle, D. Brown, L. Kidder, A. Mroué, H. Pfeiffer, M. Scheel, G. Cook, and S. Teukolsky, Phys. Rev. D 76, 124038 (2007).

[44] T. Chu, H. P. Pfeiffer, and M. A. Scheel, Phys. Rev. D 80, 124051 (2009).

[45] L. T. Buchman, H. P. Pfeiffer, M. A. Scheel, and B. Szilagyi, Phys. Rev. D 86, 084033 (2012).

[46] A. H. Mroue et al., (2013), Phys. Rev. Lett. 111, 241104 (2013).

[47] K. Arun, A. Buonanno, G. Faye, and E. Ochsner, Phys. Rev. D 79, 104023 (2009).

[48] J. Goldberg, A. MacFarlane, E. Newman, F. Rohrlich, and E. Sudarshan, J. Math. Phys. (N.Y.) 8, 2155 (1967).

[49] L. E. Kidder, Phys. Rev. D 52, 821 (1995).

[50] L. Blanchet, A. Buonanno, and G. Faye, Phys. Rev. D 74, 104034 (2006).

[51] Y. Pan, A. Buonanno, R. Fujita, E. Racine, and H. Tagoshi, Phys. Rev. D 83, 064003 (2011).

[52] E. Barausse, A. Buonanno, S. A. Hughes, G. Khanna, S. O'Sullivan, and Y. Pan, Phys. Rev. D 85, 024046 (2012).

[53] A. Buonanno, L. E. Kidder, A. H. Mroue, H. P. Pfeiffer, and A. Taracchini, Phys. Rev. D 83, 104034 (2011).

[54] H. P. Pfeiffer, D. A. Brown, L. E. Kidder, L. Lindblom, G. Lovelace, and M. A. Scheel, Classical Quantum Gravity 24, S59 (2007).

[55] G. Lovelace, M. Scheel, and B. Szilagyi, Phys. Rev. D 83, 024010 (2011).

[56] A. H. Mroue and H. P. Pfeiffer, arXiv:1210.2958.

[57] M. Purrer, S. Husa, and M. Hannam, Phys. Rev. D 85, 124051 (2012).

[58] M. Purrer, M. Hannam, P. Ajith, and S. Husa, Phys. Rev. D 88, 064007 (2013).

[59] T. Damour and A. Nagar, Phys. Rev. D 76, 064028 (2007).

[60] T. Damour, B. R. Iyer, and A. Nagar, Phys. Rev. D 79, 064004 (2009).

[61] L. E. Kidder, Phys. Rev. D 77, 044016 (2008).

[62] A. Buonanno, L. Kidder, Y. Pan, and M. Scheel, (private communication). 
[63] A. Buonanno, G. B. Cook, and F. Pretorius, Phys. Rev. D 75, 124018 (2007).

[64] E. Barausse and L. Rezzolla, Astrophys. J. 704, L40 (2009).

[65] E. Berti, V. Cardoso, and C. M. Will, Phys. Rev. D 73, 064030 (2006).

[66] W. Tichy and P. Marronetti, Phys. Rev. D 78, 081501 (2008).

[67] R. O'Shaughnessy, L. London, J. Healy, and D. Shoemaker, Phys. Rev. D 87, 044038 (2013).

[68] E. Ochsner and R. O'Shaughnessy, Phys. Rev. D 86, 104037 (2012).

[69] L. S. Collaboration, LSC Algorithm Library software packages LAL, LALWRAPPER, and LALAPPS, URL http://www .lsc-group.phys.uwm.edu/lal.
[70] T. Damour, B. R. Iyer, and B. Sathyaprakash, Phys. Rev. D 57, 885 (1998).

[71] D. McKechan, C. Robinson, and B. Sathyaprakash, Classical Quantum Gravity 27, 084020 (2010).

[72] J. Abadie et al., (LIGO Scientific Collaboration and Virgo Collaboration), Phys. Rev. D 83, 122005 (2011).

[73] J. Aasi et al., (LIGO Scientific Collaboration and Virgo Collaboration), Phys. Rev. D 87, 022002 (2013).

[74] J. Abadie et al. (LIGO Scientific Collaboration and Virgo Collaboration), Phys. Rev. D 85, 102004 (2012).

[75] http://www.black-holes.org/SpEC.html.

[76] C. Loken et al., J. Phys. Conf. Ser. 256, 012026 (2010). 